\title{
Quantifying Dislocation Microstructure Evolution and Cyclic Hardening in Fatigued Face-Centered Cubic Single Crystals
}

\author{
Ahmed M. Hussein, Jaafar A. El-Awady \\ Department of Mechanical Engineering, Whiting School of Engineering, \\ Johns Hopkins University, Baltimore, MD 21218-2682, USA
}

\begin{abstract}
Discrete dislocation dynamics simulations were performed to investigate the dislocation microstructure evolution and cyclic hardening during the early stages of fatigue loading in Nickel single crystals. The effects of the crystal size and initial dislocation densities on both the mechanical response and the evolution of dislocation microstructure were quantified. Crystals having an initial dislocation density of $10^{12} \mathrm{~m}^{-2}$ and diameter less than $2.0 \mu \mathrm{m}$ do not show any dislocation density multiplication or cyclic hardening. In contrast, crystals having the same initial dislocation density and diameters larger than $2.0 \mu \mathrm{m}$ show a significant dislocation density accumulation in the form of dislocation cell-like structures, even after only a few number of loading cycles. This dislocation density accumulation was also accompanied by considerable cyclic hardening. The dislocation cell size and its wall thickness increase with increasing crystal size. With increasing dislocation density the critical crystal size, at which dislocation cell-structures form, decreases. The information theoretic entropy is utilized as a metric to quantify the extent of dislocation patterning and the formation and evolution of dislocation cell structures over time. Cross-slip was found to play a dominant role in the dislocation cell-structure formation. Further insights on the mechanisms contributing to the observed behavior are presented and discussed.
\end{abstract}

Keywords: Strengthening and Mechanisms, Fatigue, Dislocation Pattern Evolution, Crystal Plasticity, Dislocation Dynamics

Email addresses: ahussei4@jhu.edu (Ahmed M. Hussein), jelawady@jhu.edu (Jaafar A. El-Awady) Preprint submitted to JMPS

February 28, 2016 


\section{Introduction}

The need to develop and formulate reliable fatigue damage life prediction methods for metals in aerospace applications is ever-growing. Fatigue failure is considered to be one of the main causes leading to structural and mechanical systems failure [1,2]. It has been estimated that the cost of failed mechanical components due to fatigue is around $4 \%$ of the U.S. gross national product [1]. While fatigue failure has been one of the earliest failure mechanisms to be studied, a complete understanding of mechanical fatigue still does not exist $[3,4]$. The evolution of the material microstructure under cyclic loading is sensitive to the loading conditions, environmental effects, strain rate, and the material crystal structure, among other factors. This partially explains the complexity of fatigue related failures. In addition, the recent growing interest in nanocrystalline metals for MEMS applications has lead to new questions regarding the cyclic response of these materials that are yet to be answered [5]. Thus, an in depth effort to provide physical insights regarding the important deformation mechanisms active during cyclic loading is needed to develop more robust fatigue failure predictions.

The evolution of the dislocation microstructure during cyclic loading is a complicated process, where several deformation mechanisms that span multiple length and time scales are strongly interacting. Both in situ and post-mortem experiments can only provide limited information on how the dislocation microstructure evolves under highly controlled and usually idealized loading environments. Furthermore, theories that attempt to explain the microscopic behavior, especially dislocation patterning, are usually based on simplified models in which the stress triaxiality and the dislocations interactions are either neglected or modeled using ad-hoc assumptions $[6,7,8]$. However, a full consideration of the evolution of dislocations structures is essential in an effort to partially unravel the complexity of the problem.

High fidelity computer simulations at the appropriate length scales can provide better insights on details of the dislocation microstructure evolution. Discrete Dislocation Dynamics (DDD) simulations [9, 10, 11, 12], where the physics-based evolution of dislocation 
microstructures is captured, can provide such needed understanding. DDD has been extensively utilized in the past to study the effects of crystal size on its mechanical response (e.g. $[13,14]$ ), the behavior of thin films (e.g. [15]), nanoindentation (e.g. [16]), and irradiation hardening (e.g. [17]). In DDD, most physical mechanisms required to accurately replicate the evolution of dislocations are accounted for with minimal constitutive rules or empirical laws incorporated. This makes DDD a suitable choice for explicitly modeling the collective behavior of dislocations with minimum ad-hoc assumptions if any.

Discrete Dislocation Dynamics simulations have been previously used to study several aspects of cyclic loading in metals. Deshpande et. al. [18] used two-dimensional (2D) DDD to study the interaction of dislocations with pre-existing cracks under both monotonic and cyclic loading, and concluded that dislocation activities tend to be more localized around the crack tip in cyclic loading. Brinckmann et. al. [19] used 2D DDD to study the evolution of the dislocation density and stresses in a surface grain subject to cyclic loading and concluded that stress accumulation takes place even after a low number of cycles. Déprés et. al. $[20,21]$ performed an extensive three-dimensional (3D) DDD study of early cycle fatigue behavior using the simple edge-screw model [9]. These simulations were performed starting with a single Frank-Read source in cylindrical and half-dodecahedral surface grains. It was concluded that multislip loading orientations lead to more damage accumulation than single slip orientations. The interactions of screw dislocations on parallel slip planes in idealized persistent slip band channels were also investigated using 3D DDD, and the dipole passing mechanism was assessed and interpreted in terms of the fatigue saturation stress [22].

While these studies are informative, there are still many open questions on the role of crystal size and initial dislocation density on the evolution of the dislocation microstructure and mechanical response during the early stages of cyclic loading in Face Centered Cubic (FCC) metals. To address this, atomistically informed 3D DDD simulations that include key deformation mechanisms, such as physics based cross-slip mechanisms developed earlier by the authors [23], are performed and analyzed. The paper is organized as follows. In Section 2, the simulation setup is briefly presented, and a number of new analysis methods are introduced. In Section 3, the results of the DDD simulations are presented for different 
crystal sizes, initial dislocation densities, and number of loading cycles. Section 4 discusses the results in view of different dislocation mechanisms. Finally, a summary and conclusions are presented in Section 5.

\section{Computational Methods}

For the current simulations, an in-house modified version of the open source 3D DDD simulations code, ParaDiS [24], is used. To avoid any artificial off-plane dislocation climb, ParaDis was modified to enforce that dislocations will always glide on their designated slip planes. This also applies to dislocation-dislocation reactions that result from dislocation collisions and might lead to, erroneously, placing a dislocation segment off of its slip plane. Furthermore, dislocation cross-slip is an important deformation mechanism that affects the evolution of the defect microstructure and its overall mechanical response $[25,26,27,28$, 29, 30, 31]. Based on recent extensive molecular dynamics studies, three different cross-slip mechanisms have been identified, namely: bulk, surface, and intersection (repulsive and attractive) cross-slip [32, 33, 34, 35, 36]. These new mechanisms have been incorporated in the in-house version of ParaDiS by implementing an algorithm that would efficiently detect the longest possible screw-oriented dislocation chains, classify them according to the appropriate cross-slip types then identify their probability for cross-slip to occur [23]. The activation energies and activation volumes were obtained from atomistic simulations and were reported for nickel $(\mathrm{Ni})$ in [23].

The focus of the current simulations is on FCC Ni (Young's modulus $E=210 \mathrm{GPa}$, and Poisson's ratio $\nu=0.31$ ). However, the same approach can be easily extended to study other crystalline materials. While several models of dislocation interactions with grain boundaries and twin boundaries have been recently implemented in DDD [37, 38], the current simulations are limited to free standing single crystal simulations to reduce the complexities introduced by dislocation grain/twin boundary interactions. Dislocations are allowed to escape from all six surfaces of the simulation cell, mimicking the experimental case of a free standing single crystal axially loaded with soft grips at both ends, to remove any external influence on the dislocation evolution. The loading is strain controlled with fully 
reversed cyclic loading, a strain rate of $\dot{\epsilon}=200 \mathrm{~s}^{-1}$, and a total strain amplitude of $\Delta \varepsilon=0.4$ $\%$ in the multislip crystallographic [001] direction. At this strain rate and amplitude, the duration of one loading cycle is $40 \mu \mathrm{S}$. The crystal geometry is cubical with a side length varied from $D=0.5$ to $7.5 \mu \mathrm{m}$. The initial dislocation microstructure is randomly introduced as Frank-Read (FR) sources having random line directions and randomly assigned to one of the twelve FCC slip systems. The FR source length is normally distributed with a mean of $0.6 D$ and a standard deviation of $0.03 D$, irrespective of the initial dislocation density. The sources are placed on the twelve FCC slip systems with uniform probability, and the initial dislocation density is varied in the range of $\rho_{o}=10^{11}$ to $10^{14} \mathrm{~m}^{-2}$. The number of loading cycles was in the range of 2 to 80 cycles with more computationally expensive simulations running for a lower number of cycles. A number of analysis methods have been developed in this work to analyze the DDD simulation results and are discussed in the following sections.

\subsection{Dislocation Microstructure Metric}

A simple and familiar metric for the evolution of the dislocation microstructure with time is the information theoretic entropy [39], also known as the Shannon entropy, of the dislocation density distribution. For a discrete non-negative distribution, $f_{i}$, that sums to unity, Shannon entropy is a positive real number defined as

$$
S=-\Sigma_{i}^{N} f_{i} \ln \left(f_{i}\right)
$$

where $f_{i}$ is the value of the distribution at the $i^{\text {th }}$ point, and $N$ is the total number of points. Here, the simulation cell is uniformly meshed into $l \times m \times n$ elements and the total dislocation length inside each element is computed from the dislocation microstructure in that element. The distribution $f_{i}$ is then defined at the center of each element, and the entropy can be calculated using Equation (1). Before calculating the entropy, the values of the distribution $f_{i}$ are normalized by the total dislocation length so that $\Sigma_{i}^{N} f_{i}=1$.

From Equation (1), it can be shown that the entropy of a uniform distribution is maximum, while that of a singleton (the distribution where one value of the random variable has a probability of one and all other values have probabilities of zero) is zero. In terms of 
dislocation distributions, a crystal with all dislocations concentrated at a single point will have a zero entropy, while that where the dislocations are uniformly distributed will have a maximum entropy, which in this case will equal $S_{\max }=\ln (l \times m \times n)$. The simulations start with a number of Frank-Read sources, which mimic the case of a singleton, although not perfectly, and it evolves over time into bigger regions that have a uniform dislocation density. By calculating the relative entropy, defined to be the ratio of the dislocation density distribution entropy to the maximum possible entropy, it is possible to measure how far the system has evolved in terms of forming dislocation structures. Simulations that show no relative entropy change maintain their initial configuration of random dislocation distributions, while those that show an increase in their relative entropy show the development of dislocation structures.

\subsection{Quantifying the Dislocation Microstructure Size}

As will be discussed in Section 3, in the current simulations dislocation cell-like structures evolve and it is important to quantify their average size. Since these cells are not geometrically ideal, a simple method, such as fitting ellipsoids to the low dislocation density cell interior, will be sensitive to the irregularities of the dislocation structure and is not expected to give good accuracy.

Alternatively, the method used here builds on the work of Zaiser et. al. [40], which was based on image analysis of 2D transmission electron microscopy (TEM) micrographs of dislocation cell structures to estimate a number of fractal dimensions, and subsequently quantify dislocation cell sizes. In the 3D setting of DDD, at any particular time the simulation cell is meshed into elements and the dislocation density is calculated in each element. All elements with a dislocation density less than a threshold value of $\rho_{t h}=10^{14} \mathrm{~m}^{-2}$ are neglected. Subsequently, the remaining elements that correspond to the interior of a dislocation wall are represented as a number of points, $p_{i}$, and their distribution gives the general shape of the dislocation walls. The simulation cell is then divided one more time into cubes $c_{i}$ of edge length $L$. The total number of points, $p_{i}$, that are contained within each cube are summed and then the number of cubes, $n$, that have at least one point is recorded. The 
process is repeated for cubes of different edge lengths, $L$, to get the final distribution $n(L)$. A 2D schematic representation of the cube counting process is shown in Figure 1(a).
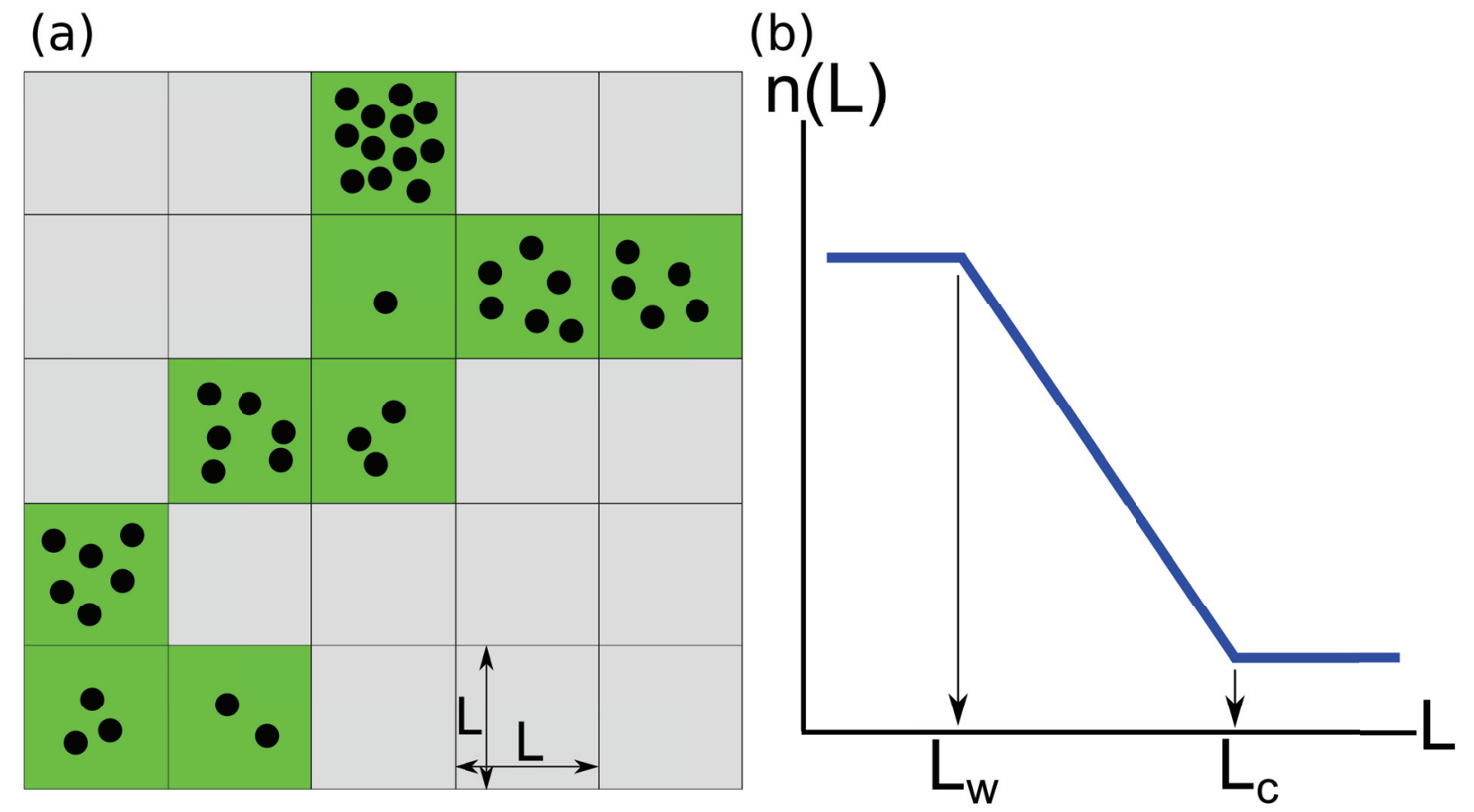

Figure 1: (a) A 2D schematic of the box-counting process. The black circles are the centers of the cells having a dislocation density higher than the threshold dislocation density. The squares (cubes in 3D) are equal in size with a side length $L$. The green squares contain at least one high dislocation density point. In this example $n(L)=9$. (b) A typical plot of $n(L)$ versus $L$ showing the transitions at which the wall thickness, $L_{w}$, and the cell size, $L_{c}$, can be quantified.

The distribution, $n(L)$, is then used to estimate the dislocation cell size, $L_{c}$, and dislocation wall thickness, $L_{w}$ [40]. By plotting $n(L)$ versus $L$, the distribution is usually constant at low and high $L$ values and decreases for intermediate values of $L$, as shown in Figure 1(b). For small values of $L$, increasing $L$ does not result in a significant change in $n(L)$ because the cube edge length is not yet equal to the dislocation wall thickness. When $L$ is equal to the dislocation wall thickness, $L_{w}$, there is one cube that spans the entire wall thickness. Any further increase in the cube's edge length will cause the cubes at the cell corners to cover more dislocation wall regions, which consequently reduces the number of the cubes 
needed to cover the entire dislocation wall (i.e. $L>L_{w}, n(L)$ decreases). As $L$ is increased further, each cube covers increasingly growing regions of the dislocation wall structure and fewer cubes are needed, which maintains the decreasing monotonicity of $n(L)$. When $L$ becomes large enough that one cube spans an entire dislocation cell, (i.e. $L=L_{c}$ ), any further increase in $L$ will not result in any change in $n(L)$, since each cube now covers about one dislocation cell and its surrounding dislocation walls. The dislocation wall thickness and dislocation cell size determined by this method are approximate values and they are sensitive to several factors such as the threshold dislocation density and how uniform the dislocation structure is. Nevertheless, this gives a first order approximation for the characteristic length scales of the dislocation microstructure.

\section{Results}

The engineering stress versus engineering strain curves under cyclic loading are shown in Figure 2 from representative simulations having different crystal sizes and initial dislocation densities. For smaller crystal sizes, the overall strength is higher and it decreases as the crystal size increases. The successive drops in the stress after yield correspond to plastic strain bursts, which are less pronounced at higher dislocation densities or larger crystal sizes. At lower dislocation densities and smaller crystal sizes, a larger scatter in the flow stress is observed from one loading cycle to another, and this scatter decreases with increasing dislocation density. Furthermore, for the smallest crystals having size $D=0.5 \mu \mathrm{m}$ and initial dislocation density of $\rho_{o}=10^{14} \mathrm{~m}^{-2}$ display no sharp yield points, and the stressstrain curve transitions smoothly in a nonlinear manner from elastic loading to plastic flow. This is due to plastic activity initiating early, leading to continuous softening. This is also observed for the $D=2.0 \mu \mathrm{m}$ crystals having an initial dislocation density of $\rho_{o}=10^{13} \mathrm{~m}^{-2}$.

Figure 3 shows the crystal strength and dislocation density versus simulation time from representative simulations of each crystal size, all having an initial dislocation density of $\rho_{0}=1 \times 10^{12} \mathrm{~m}^{-2}$. Three observations can be made here. First, the strength decreases with increasing crystal size. Second, for the number of cycles simulated, the dislocation density in the crystals having sizes $D \leq 1.0 \mu \mathrm{m}$ remains constant even with increasing number of cycles. 
The almost regular spikes in the dislocation density evolution, which coincide with the stress drops, correspond to the activation of one, or at most a few, dislocation sources and their subsequent escape from the crystal surface. The analysis of surface roughness evolution due to the escape of these dislocation segments has been reported in [41]. On the other hand, the density multiplies significantly faster in crystals having sizes $D \geq 2.0 \mu \mathrm{m}$, even after just a few cycles. These results indicate that the dislocation density multiplication factor is size dependent, with the dislocation density multiplication increasing with increasing crystal size. Finally, strong cyclic hardening is also observed in crystals having sizes $D \geq 5.0 \mu \mathrm{m}$, and almost none are observed for smaller crystals for the number of cycles simulated.

In addition to the effect of the crystal size, the initial dislocation density plays an important role in controlling both the overall response as well as the evolution of the dislocation microstructure. Figure 4 shows representative engineering stress and dislocation density curves as a function of the simulation time for two different crystal sizes starting with three initial dislocation densities each. In both crystal sizes, the overall strength decreases with increasing initial dislocation density, which agrees with the predictions of the generalized size-dependent Taylor-strengthening law [14]. It is also observed that for $D=0.75 \mu \mathrm{m}$, the dislocation density does not change much, for the number of cycles simulated, irrespective of the initial dislocation density. This indicates that the dislocation multiplication and escape rates are equal in smaller crystals. For the $D=2.0 \mu \mathrm{m}$, the dislocation density is observed to significantly increase for simulations with higher initial dislocation densities, while it remains mostly constant for simulations with lower initial dislocation densities.

The initial and final dislocation microstructures for crystals of different sizes and an initial dislocation density of $\rho_{o}=10^{12} \mathrm{~m}^{-2}$ are shown in Figure 5. Furthermore, the evolution of the dislocation microstructure in a $D=2.0 \mu \mathrm{m}$ having an initial dislocation density of $\rho_{o}=10^{13} \mathrm{~m}^{-2}$, is shown in supplementary movie $\mathrm{I}$, while the $3 \mathrm{D}$ view of the dislocation microstructure in a $D=5.0 \mu \mathrm{m}$ crystal having an initial dislocation density of $\rho_{o}=10^{12}$ $\mathrm{m}^{-2}$ is shown in supplementary movie II. Smaller crystals having $D<2.0 \mu \mathrm{m}$ do not exhibit any microstructure buildup, even after 80 cycles, and the final microstructure has the same random distribution features as the initial microstructure. Larger crystals having $D \geq 5.0$ 
$\mu \mathrm{m}$, however, show the formation of regions of high dislocation densities surrounding regions of low dislocation densities, starting after only two cycles. These emerging dislocation microstructures resemble the experimentally observed dislocation cells with dislocation dense walls and less dislocation dense interiors $[42,43]$. The high dislocation density regions are dominated by immobile dislocations, which act as forest dislocations that pin mobile dislocations. It is also observed that many dislocations in the wall regions originate from the multiplication of a few sources from the initial dislocation network due to successive intersection cross-slip events. The high local stresses resulting from the high dislocation densities around the walls reduces the activation barrier for cross-slip further, which subsequently leads to increasing the dislocation wall's local dislocation density.

Figure 6 shows the initial and final microstructure for $D=2 \mu \mathrm{m}$ crystals having different initial dislocation densities. For low initial dislocation densities $\left(\rho_{o} \leq 10^{11} \mathrm{~m}^{-2}\right)$, no dislocation buildup is observed. As the initial dislocation density increases, dislocations start to form dislocation rich regions through dislocation pinning and multiplication. These dislocation rich regions, however, do not have the same geometric features as dislocation cell structures.

It is clear from Figures 5 and 6 that both the crystal size and the initial dislocation density play an important role in the subsequent evolution of the dislocation microstructure. For the same initial dislocation density, simulations of smaller crystals show no dislocation cell structure formation, while clear dislocation cell structures are observed for simulations of larger crystals. On the other hand, for the same crystal size, simulations with low initial dislocation densities show negligible dislocation cell structure formation even after 80 loading cycles, while simulations with higher initial dislocation densities show the formation of dislocation cell structures even after a few cycles. Nevertheless, it could be expected that a similar dislocation buildup would take place for smaller crystals with higher initial dislocation density, or larger crystals with lower initial dislocation densities, after a considerably larger number of cycles. This is however not attainable in the current simulations since the number of degrees of freedom involved makes simulations of higher number of cycles unachievable with the currently available computational power. 
The dislocation density relative entropy (DDRE) versus time for crystals of different sizes an initial dislocation densities is shown in Figure 7. Larger crystals having $D \geq 5$ $\mu \mathrm{m}$ and a low initial dislocation density of $\rho_{o}=10^{11} \mathrm{~m}^{-2}$ show a continuous increase in DDRE, indicating the formation of a dislocation cell structure. This is opposed to smaller crystals having sizes $D \leq 2.0 \mu \mathrm{m}$ and the same initial dislocation density, even though they are simulated for a higher number of cycles. For simulations with a higher initial dislocation density of $\rho_{o}=10^{12} \mathrm{~m}^{-2}$, the larger crystals, having sizes $D \geq 5.0 \mu \mathrm{m}$ show an earlier increase in DDRE, which indicates that dislocation cell structure formation starts even sooner. In addition, crystals having sizes $D=2.0 \mu \mathrm{m}$ and the same initial dislocation density show an increase in DDRE and dislocation cell structure buildup only after a larger number of cycles. However, smaller crystals having sizes $D<2.0 \mu \mathrm{m}$ and this same initial dislocation density do not show any dislocation cell structure formation and the DDRE oscillates around a low value even for a higher number of cycles. For simulations with an initial dislocation density of $\rho_{o}=10^{13} \mathrm{~m}^{-2}$, crystals having sizes as small as $D=1.0 \mu \mathrm{m}$ show some slow DDRE increase, which indicates that a more pronounceable dislocation cell structure formation might develop if the simulations are continued for a higher number of cycles. In addition, crystals having size $D=2 \mu \mathrm{m}$ now show a significant DDRE increase and a corresponding dislocation cell structure formation much earlier in the simulations. Finally, for simulations with an initial dislocation density of $\rho_{o}=10^{14} \mathrm{~m}^{-2}$, no dislocation cell structure formation or increase in DDRE are observed for simulated crystals having $D<1.0 \mu \mathrm{m}$ up to the number of simulated cycles. However, at this high density, the DDRE oscillates in a manner similar to the oscillations observed for larger crystals at lower densities before they showed any dislocation cell structure formation (e.g. $D \leq 2.0 \mu \mathrm{m}$ and $\rho_{o}<10^{12} \mathrm{~m}^{-2}$, which suggests that dislocation cell structure formation could take place if these crystals were simulated for a higher number of cycles or if the initial dislocation density was higher. These observations suggest the existence of a size-dependent critical dislocation density beyond which dislocation cell structure formation takes place. It also should be noted that the monotonic increase in the DDRE observed for simulations of larger crystals indicates that the load reversal does not break the dislocation cell structures that 
have formed.

It should be noted that the computational cost of these simulations depends on the number of degrees of freedom, which in turn depends on the crystal size and the initial dislocation density. Unlike most computational methods, the number of degrees of freedom in DDD simulations varies over the course of the simulation. As the dislocation density increases through dislocation glide or cross-slip, more dislocation nodes are added to the simulations in order to better accurately capture the dislocation geometry. As dislocations mutually annihilate or escape from the surface, dislocation nodes are removed from the system. This adds a third factor which affects the computational cost of these simulations, that is, the complexity of the dislocation reactions and the subsequent dynamics of dislocations. Unfortunately, this latter factor cannot be easily quantified. In the current simulations, the wall-clock run time per loading cycle varies from about 500 processor-hours for the small simulations $\left(D=0.5 \mu \mathrm{m}\right.$ and $\left.\rho_{o}=10^{12} \mathrm{~m}^{-2}\right)$ to about 730,000 processor-hours for the large simulations $\left(D=5.0 \mu \mathrm{m}\right.$ and $\left.\rho_{o}=10^{12} \mathrm{~m}^{-2}\right)$. The computational load is almost uniform for the smaller simulations such that one can simulate the same number of loading cycles per processor-hour. On the other hand, the computational cost increases exponentially for larger simulations such that the first few cycles take a fewer number of processor-hours to complete while the later ones take much longer to finish. 
(a)

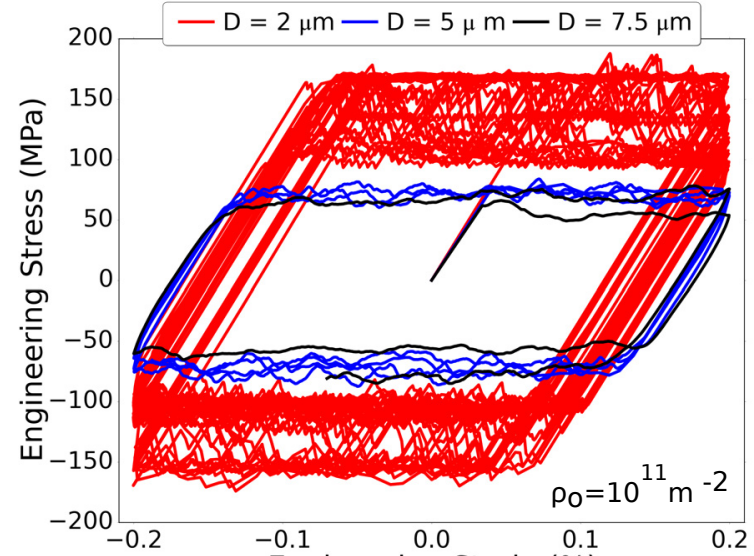

(c)

Engineering Strain (\%)

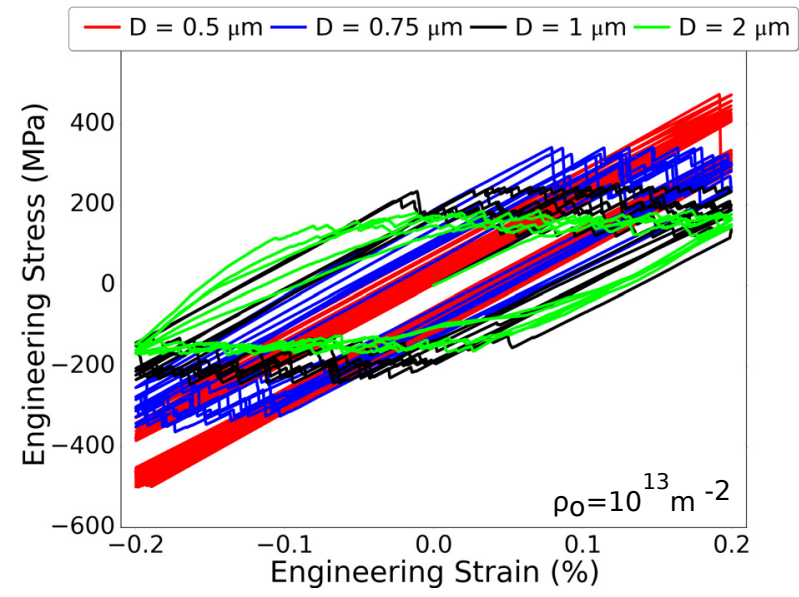

(b)

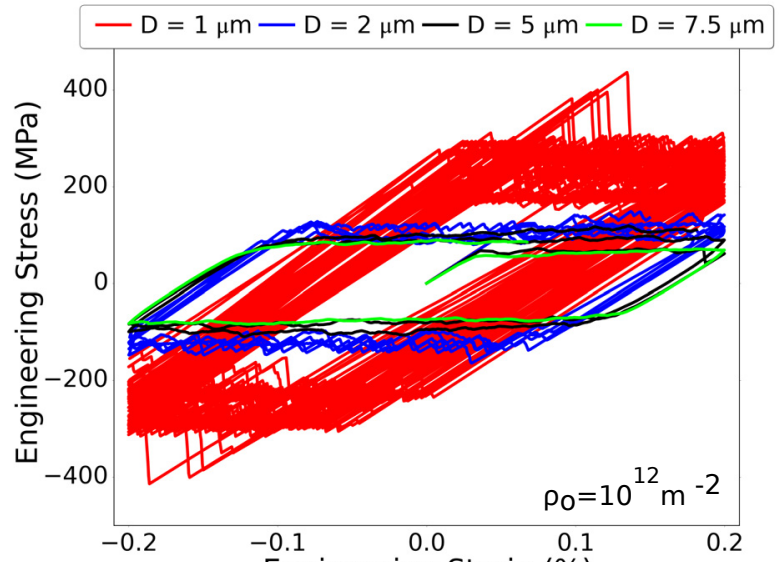

(d)

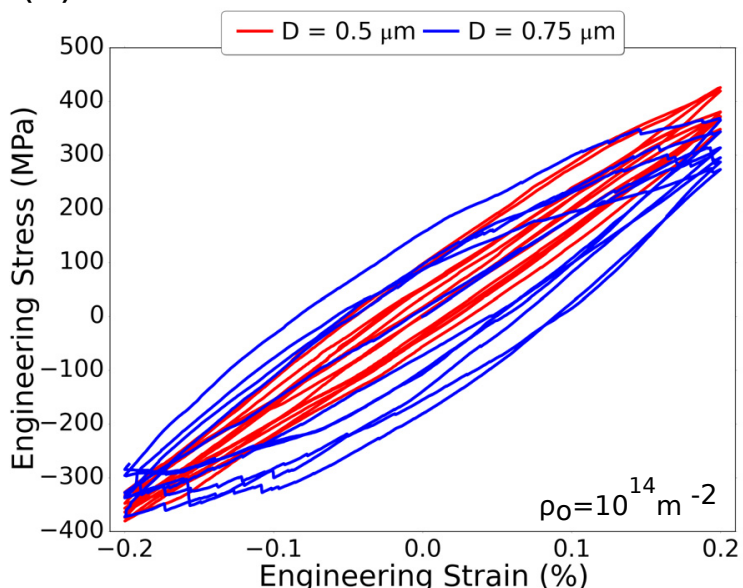

Figure 2: The engineering stress-strain curves from representative simulations having different crystal sizes and grouped by the initial dislocation density: (a) $\rho_{o}=10^{11} \mathrm{~m}^{-2}$; (b) $\rho_{o}=10^{12} \mathrm{~m}^{-2}$; (c) $\rho_{o}=10^{13} \mathrm{~m}^{-2}$; and (d) $\rho_{o}=10^{14} \mathrm{~m}^{-2}$. 


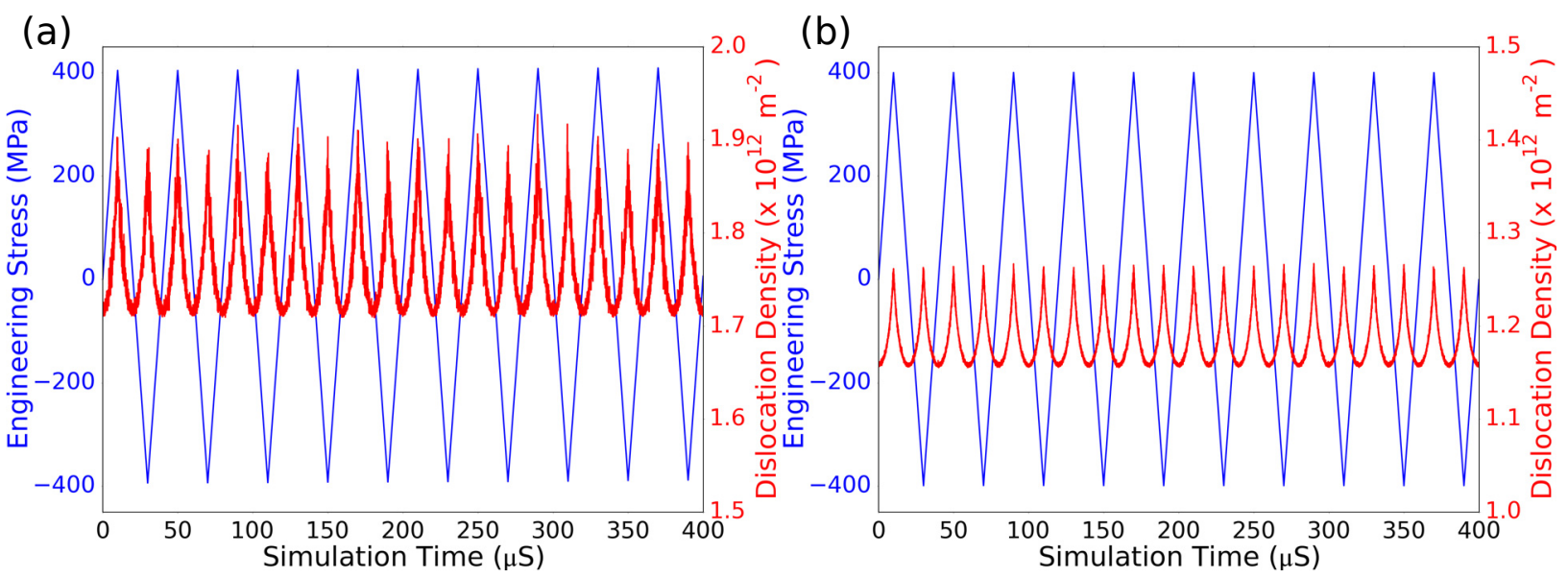

(c)

$(\mathrm{d})$

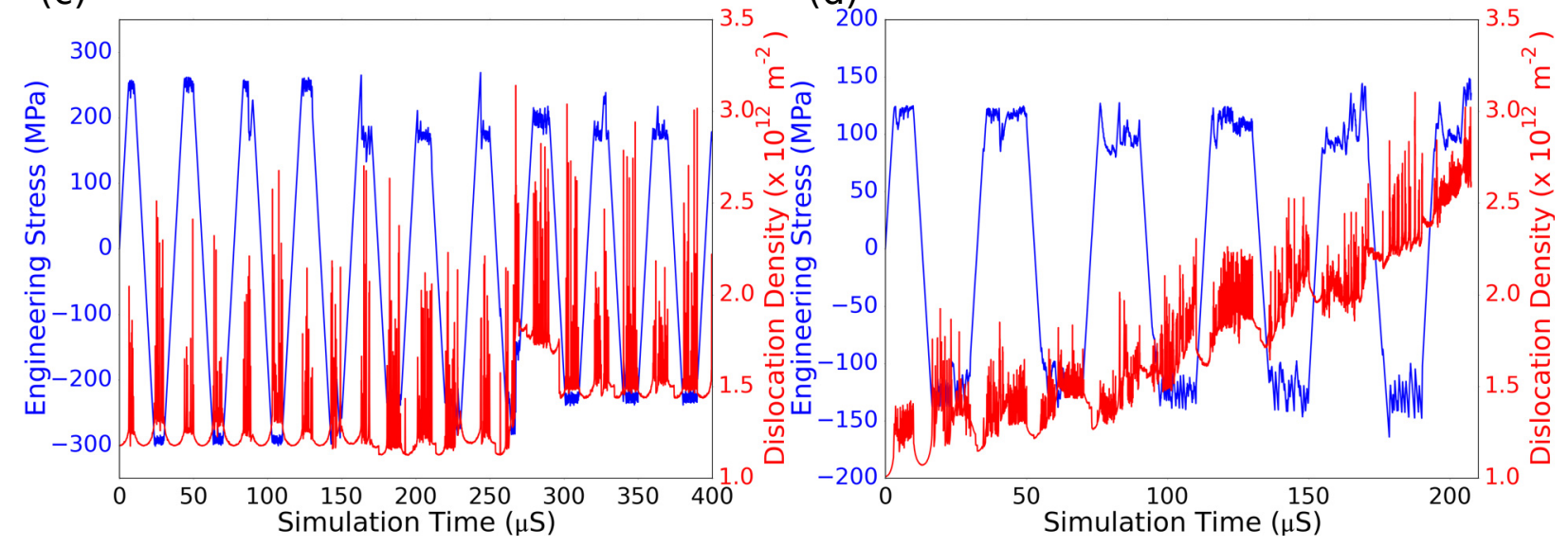

(e)

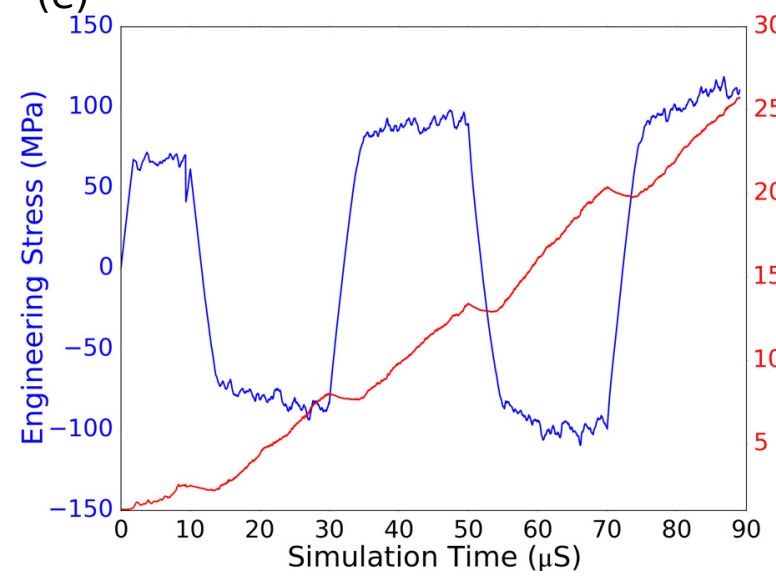

(f)

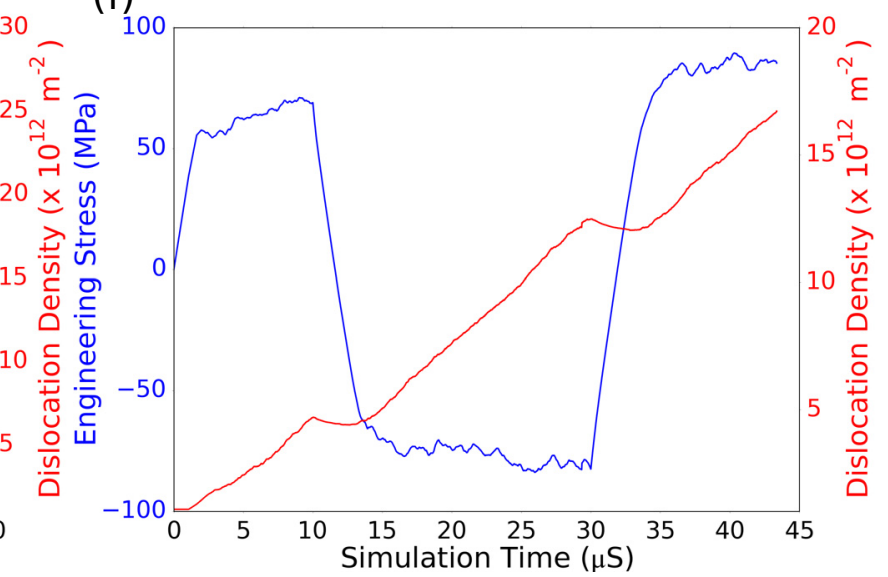

Figure 3: The engineering stress and dislocation density as a function of simulation time for simulations with an initial dislocation density of $\rho_{o}=10^{12} \mathrm{~m}^{-2}$ and crystal sizes: (a) $0.5 \mu \mathrm{m}$; (b) $0.75 \mu \mathrm{m}$; (c) $1.0 \mu \mathrm{m}$; (d) $2.0 \mu \mathrm{m}$; (e) $5.0 \mu \mathrm{m}$; and (f) $7.5 \mu \mathrm{m}$. 


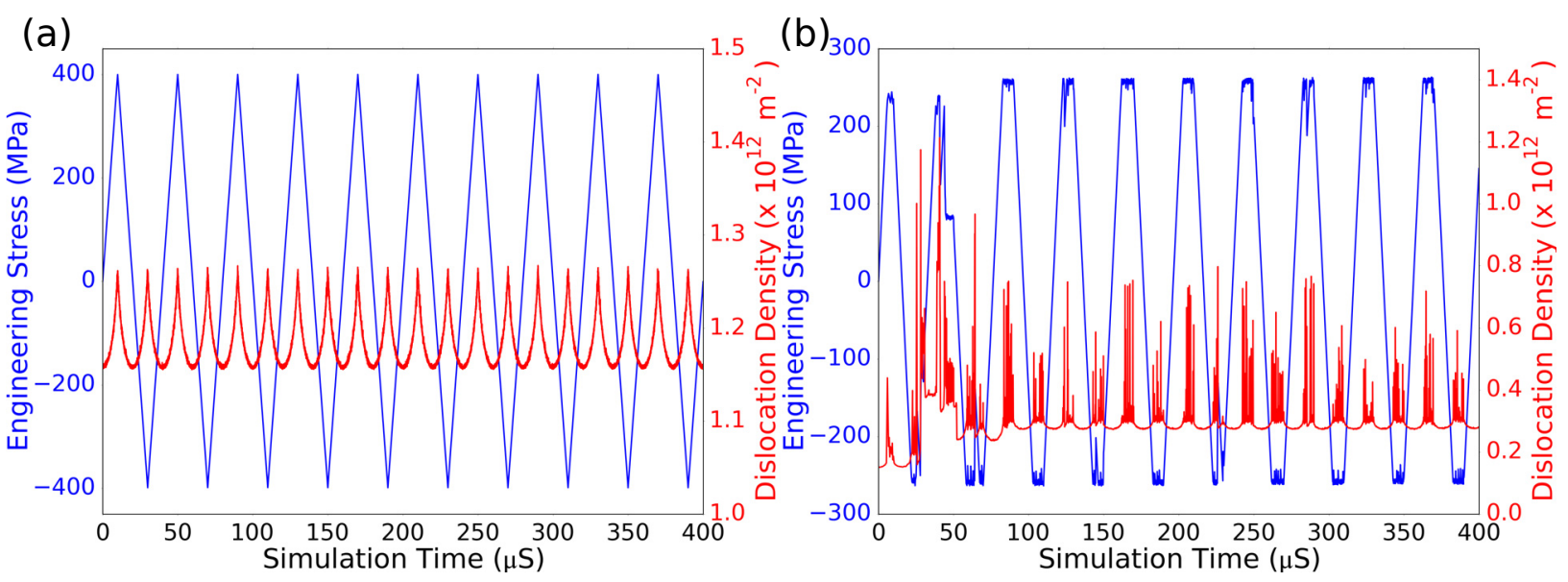

(c)

(d)

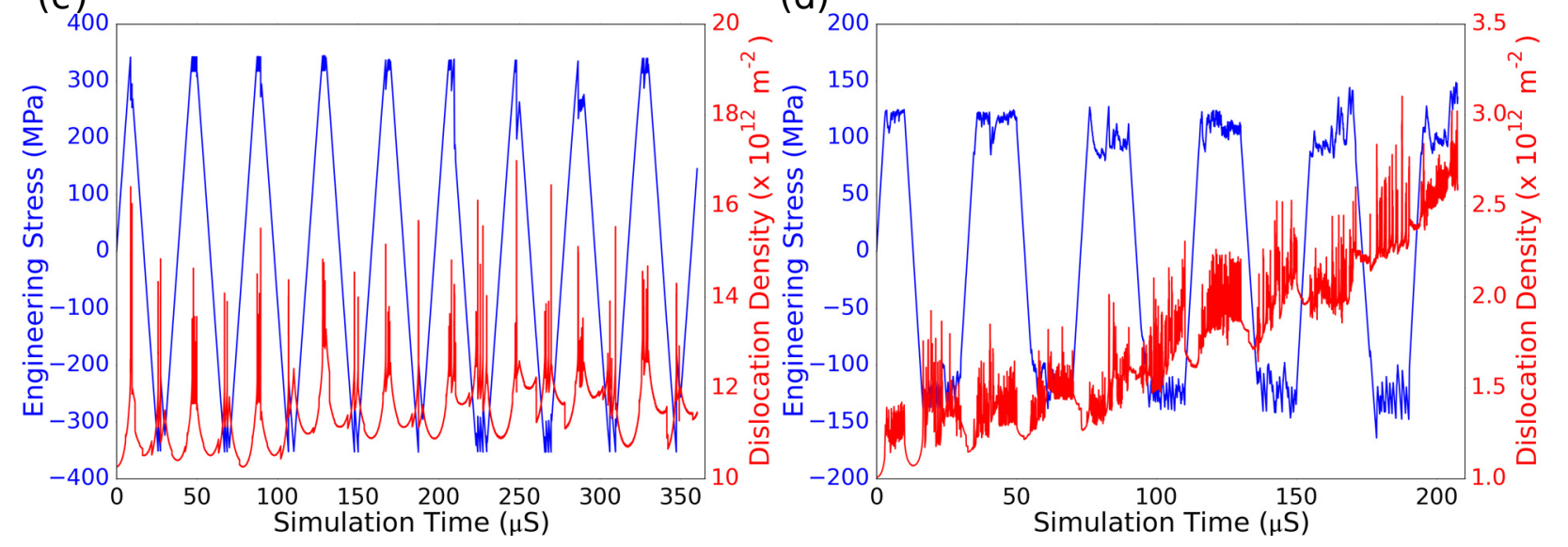

(e)

(f)

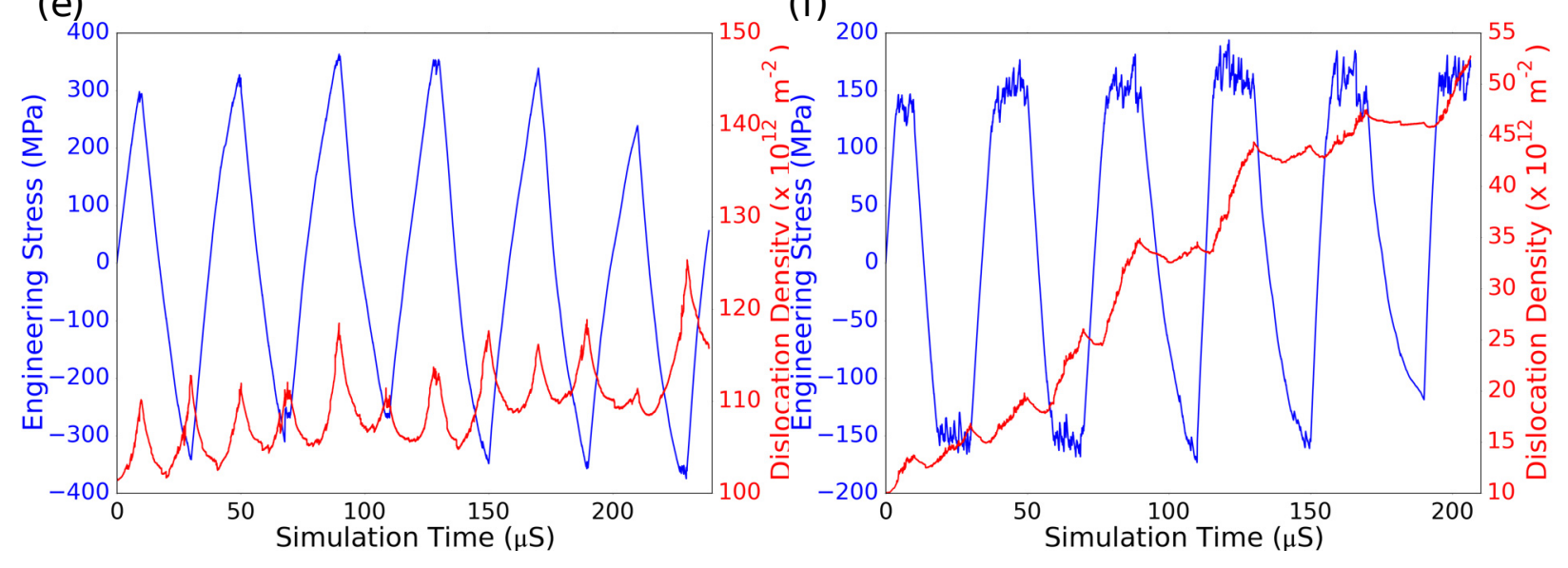

Figure 4: The engineering stress and dislocation density as a function of simulation time for crystals having size $D=07.5 \mu \mathrm{m}$ and: (a) $\rho_{o}=10^{12} \mathrm{~m}^{-2}$, (b) $\rho_{o}=10^{13} \mathrm{~m}^{-2}$, (c) $\rho_{o}=10^{14} \mathrm{~m}^{-2}$; and crystals having size $D=2 \mu \mathrm{m}$ and: (d) $\rho_{o}=10^{11} \mathrm{~m}^{-2}$, (e) $\rho_{o}=10^{12} \mathrm{~m}^{-2}$ and (f) $\rho_{o}=10^{13} \mathrm{~m}^{-2}$. 
(a)

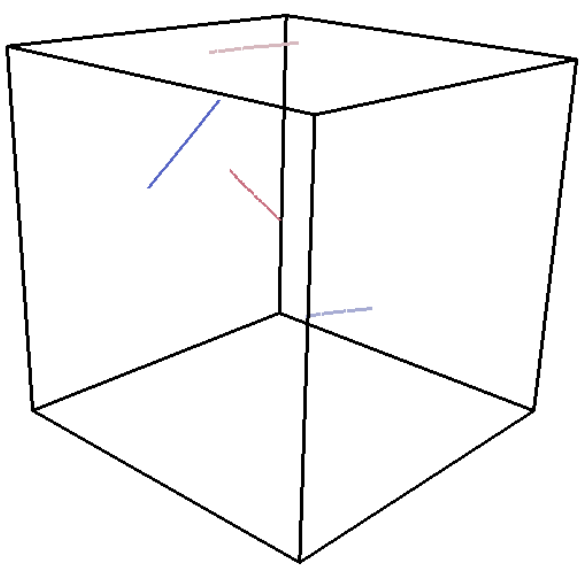

(b)

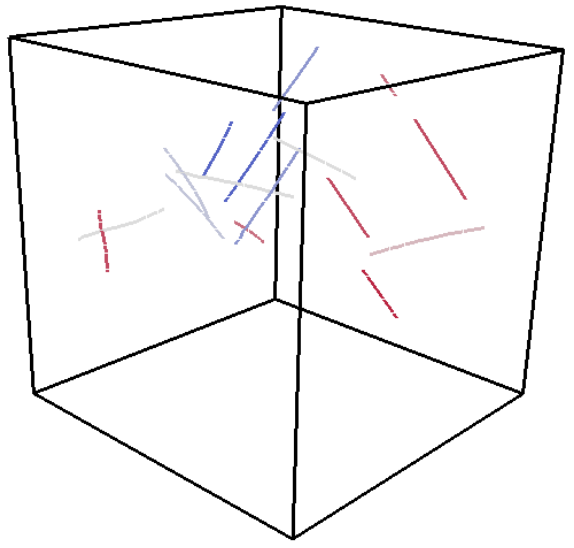

(c)

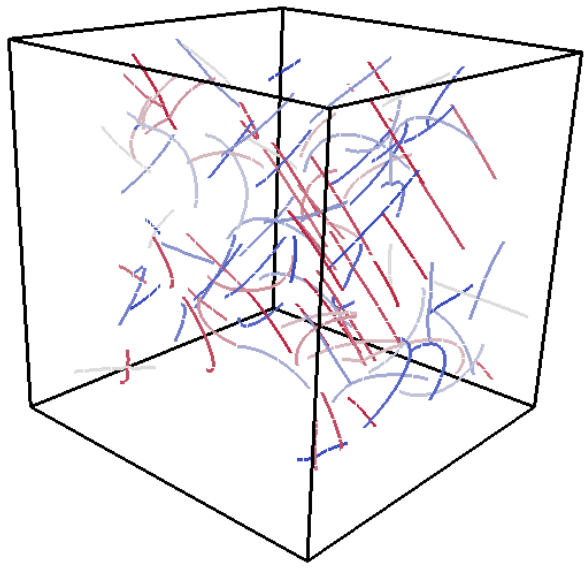

After 80 loading cycles

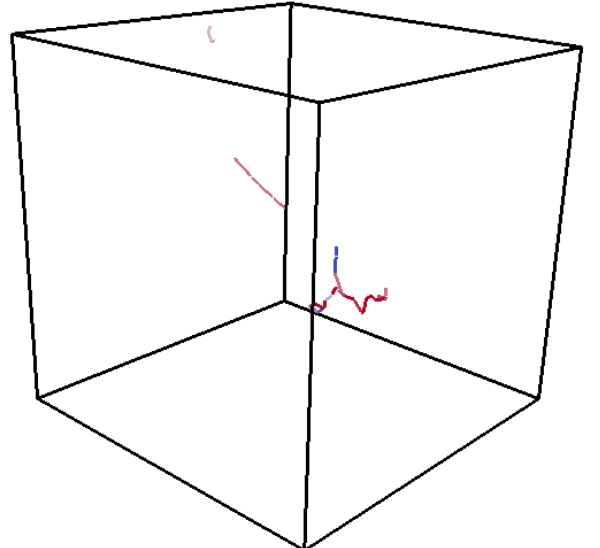

After 5 loading cycles

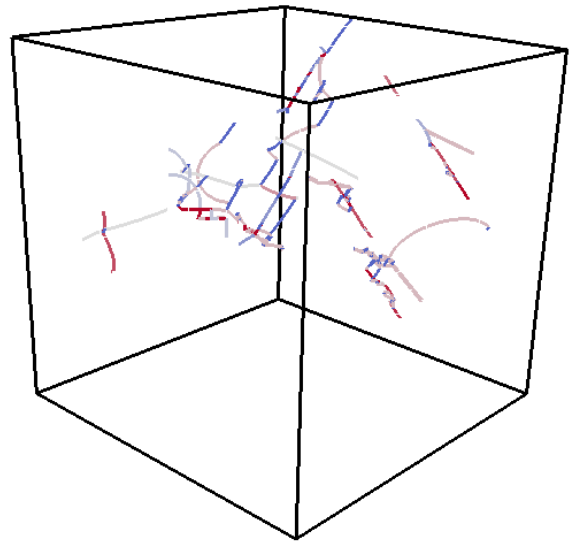

After 2 loading cycles

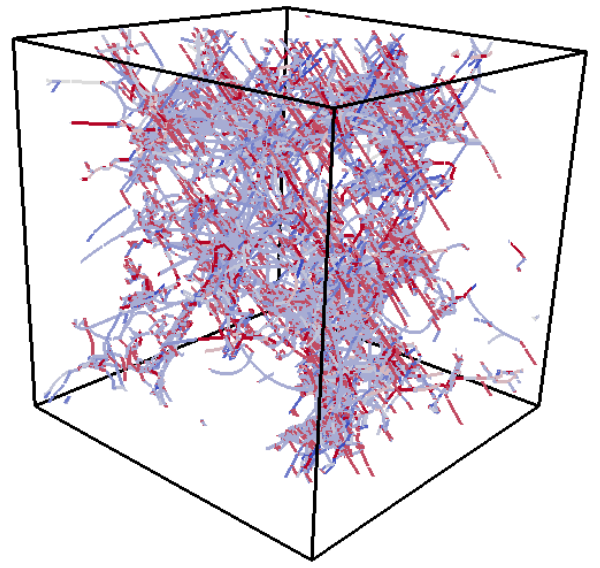

Figure 5: The initial and final dislocation microstructures for simulations having an initial dislocation density of $\rho_{o}=10^{12} \mathrm{~m}^{-2}$ and sizes: (a) $D=1.0 \mu \mathrm{m}$; (b) $D=2.0 \mu \mathrm{m}$; and (c) $D=5.0 \mu \mathrm{m}$. 
(a)

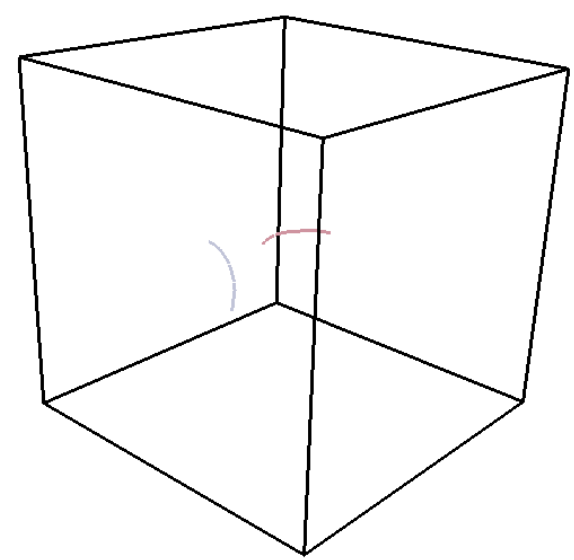

(b)

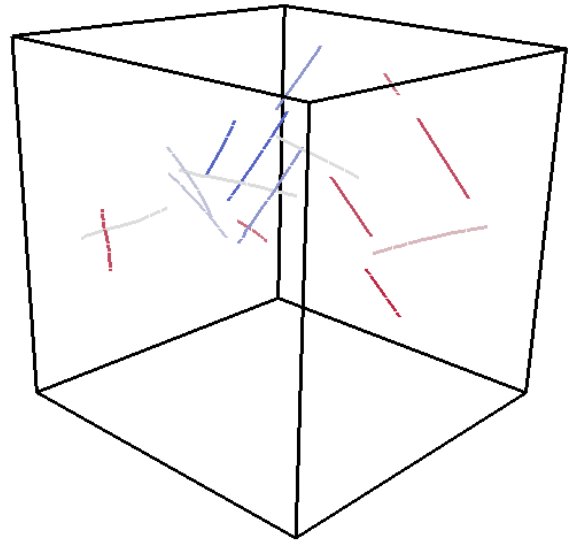

(c)

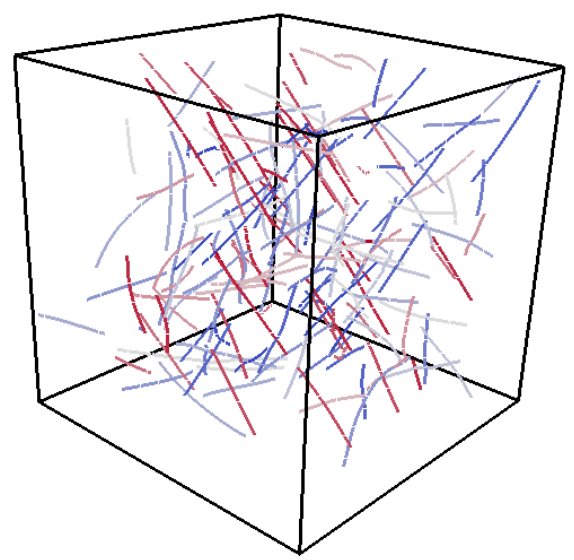

After 36 loading cycles

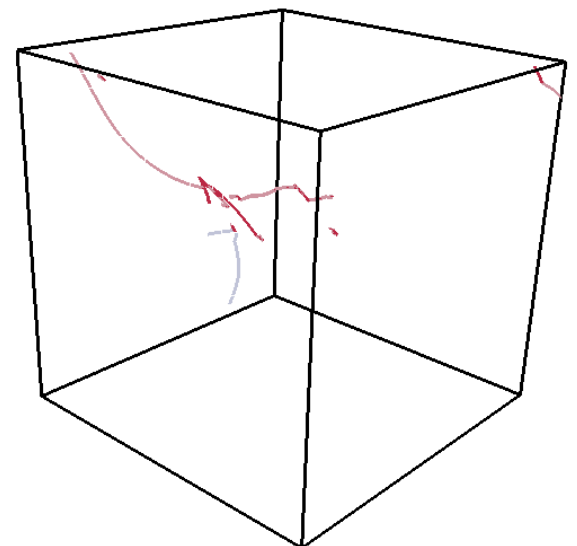

After 5 loading cycles

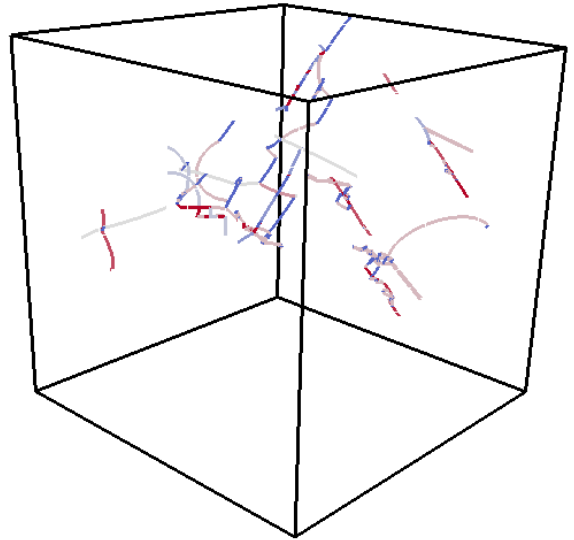

After 5 loading cycles

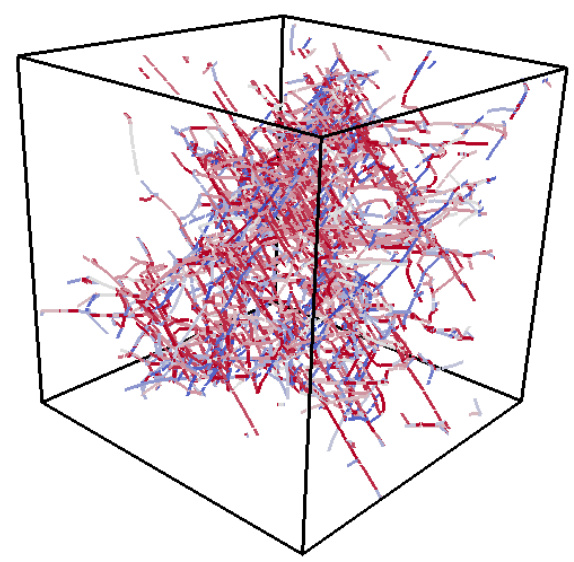

Figure 6: The initial and final dislocation microstructures for simulations of crystals having sizes $D=2.0$ $\mu \mathrm{m}$ and: (a) $\rho_{o}=10^{11} \mathrm{~m}^{-2}$; (b) $\rho_{o}=10^{12} \mathrm{~m}^{-2}$; and (c) $\rho_{o}=10^{13} \mathrm{~m}^{-2}$. 

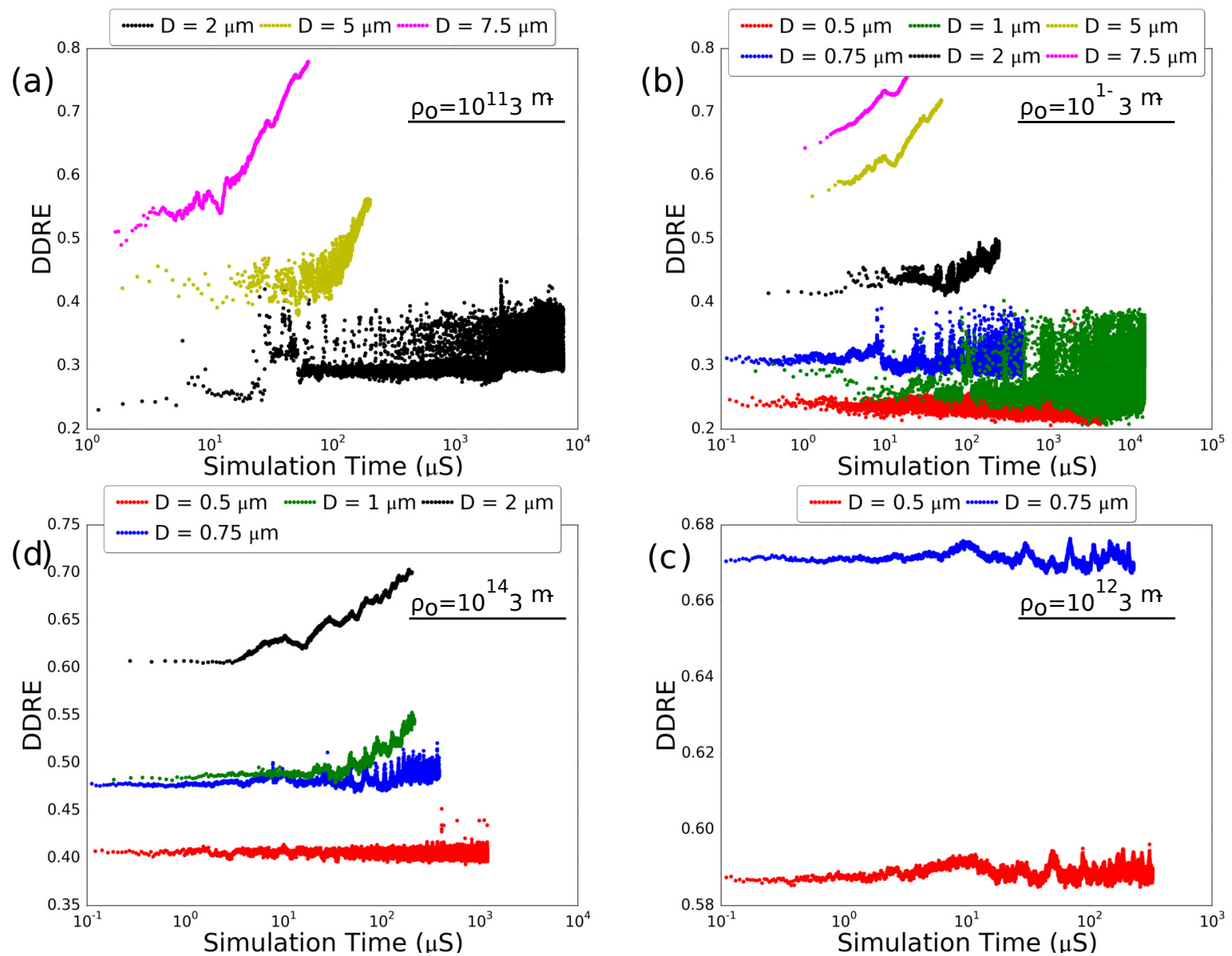

Figure 7: The dislocation density relative entropy (DDRE) versus simulation time for different crystal sizes and: (a) $\rho_{o}=10^{11} \mathrm{~m}^{-2}$; (b) $\rho_{o}=10^{12} \mathrm{~m}^{-2}$; (c) $\rho_{o}=10^{13} \mathrm{~m}^{-2}$; and (d) $\rho_{o}=10^{14} \mathrm{~m}^{-2}$. 


\section{Discussion}

\subsection{Dislocation Density Evolution}

Figure 8(a) shows the ratio of the dislocation density at the end of each cycle to the initial dislocation density versus the number of loading cycles for different crystal sizes and initial dislocation densities. Each data point is an average of three different simulations with varying initial random dislocation distributions. It is observed that larger crystals show a high rate of dislocation density multiplication, which is a direct result of significant dislocation cross-slip events, and in particular intersection cross-slip at the collision points of dislocations on non-parallel planes, as shown in Figure 8(b). Dislocations glide, collide, then cross-slip in a repeated manner, which leads to storing more dislocations within the crystal rather than their escape. The dislocation density multiplication can reach up to 75 times after only two loading cycles for the larger crystals. This is the main reason these simulations are computationally challenging to run for larger numbers of loading cycles. The increase in the number of degrees of freedom over time makes the simulation computationally demanding and it becomes impractical to continue the simulations further.

A similar mechanism was suggested by Déprés et. al. [20] to explain the formation of dislocation microstructures, however, they had only accounted for the traditional bulk cross-slip mechanism. In an earlier study, the current authors showed that the number of new dislocation sources generated during monotonic loading due to intersection cross-slip increases considerably with increasing crystal size [23]. On the other hand, when bulk cross-slip is only accounted for the dislocation multiplication is significantly slower and no considerable dislocation buildup is expected during early stages of the deformation. This latter case is in contradiction with experimental observations showing dislocation cell structure formation starting even at low strain levels $[44,45,46]$.

The dislocation density evolution per slip system versus time is shown in Figure 9 for some representative simulations. As shown in Figure 9(a), in smaller crystals having $D \leq 2.0 \mu \mathrm{m}$, the dislocation density remains relatively constant, with some fluctuations on all slip systems. This is typical for all crystals in Figure 8(a) that show little or no density multiplication. 
When significant dislocation density multiplication is observed (i.e. for larger crystals or higher initial dislocation densities), two dislocation density evolution trends can be identified. In particular, one pair (Figure 9(c)), or multiple pairs (Figure 9(b) and (d)) of slip systems show a significant density increase, while other slip systems show negligible dislocation density buildup. The slip systems of each pair share a common Burgers vector, which indicate that the increase in dislocation density on these slip systems is due to continuous cross-slip of dislocations back and forth from one slip plane to the other. The number of slip system pairs that are active depends on the number of sources that have the potential to multiply and cross-slip. This number increases with increasing crystal size and/or the initial dislocation density. Hence, for larger crystals (Figure $9(\mathrm{~d})$ ) or crystals with a higher initial dislocation density (Figure 9(b)), multiple pairs activate, in contrast with lower initial dislocation density crystals (Figure 9(c)) where only one pair is active. Smaller crystals (Figure 9(a)) do not show such a behavior because dislocations can escape from the crystal surface shortly after becoming active and before getting a chance to be engaged in any intersection cross-slip activity.

\subsection{Flow Strength and Cyclic Hardening}

Early cyclic strain hardening has been experimentally studied in pure copper and Nickel [47, 48, 49]. Morrison et. al. [49] observed that cyclic hardening rates are higher for crystals oriented for multislip due to the interactions of dislocations on different slip systems. They also showed that the buildup of dislocation microstructures, particularly dislocation cell structures, goes hand in hand with cyclic hardening. In the current DDD simulations, larger crystals showed early cyclic hardening, which is attributed to the buildup of large immobile dislocation assemblies as discussed in Section 4.3. These dislocation walls reduce the mean free path of mobile dislocations in the crystal. The relative strength at the end of different loading cycles to the strength at the end of the first quarter cycle for all the simulated cases is shown in Figure 8(c). Each data point is the average of three simulations with the same configuration but different random initial dislocation distributions. Smaller crystals that do not form any dislocation cell structures do not show any cyclic hardening 
even up to 80 cycles. On the other hand, larger crystals that show the development of dislocation cell structures show up to $40 \%$ increase in the yield strength after only two loading cycles. Experimental observations of copper single crystals fatigued in a multislip orientation suggest that cross-slip may play an important role in early cyclic hardening [50]. Those experimental suggestions along with the results in Figure 8(b), which indicate that cross-slip is frequent in larger crystals that show cyclic hardening, show the agreement between the current DDD simulations and the experimental observations when it comes to predicting cyclic hardening response.

A single crystal shear strength in dislocation mediated plasticity is typically expressed as $\tau=b k / \lambda$, where $k \in[0,1)$ is a strength coefficient, and $\lambda$ is the effective (or mean) dislocation source length in the crystal. Recent DDD simulations of size effects during monotonic loading of microcrystals [14] have shown that the effective source length below a critical dislocation density varies with crystal size as $\lambda=b D \sqrt{\rho} / \beta$, while above that critical density it is size independent with $\lambda=\alpha / \sqrt{\rho}$. Thus, the crystal strength was shown to follow:

$$
\frac{\tau}{\mu}=\frac{\beta}{D \sqrt{\rho}}+\alpha b \sqrt{\rho}
$$

where $\tau$ is the crystal strength, $b$ is the Burgers vector magnitude, and $\alpha=0.57$ and $\beta=1.76 \times 10^{-3}$ are two dimensionless constants. It has been shown that Equation (2) is in excellent agreement with both DDD simulations and experiments [14]. Figure 10 shows $\frac{\tau}{\mu} \sqrt{D}$ versus $D \rho$ from all the simulations at the end of each quarter cycle, as well as the predictions from Equation (2) for comparison. While, as expected, there is a high scatter in the simulation results, the simulations agree on average with the predictions of Equation (2). The predictions from Equation (2) are slightly lower than the simulation data, which is mainly because the constants $\alpha$ and $\beta$ were computed based on the initial dislocation density [14], while the simulation results in Figure 10 are the instantaneous strength and dislocation density at the end of each quarter cycle. For larger crystal sizes or higher initial dislocation densities, the slope of the simulation data in Figure 10 agrees well with the slope 
predicted from Equation (2) for the same range of $\rho D$. For smaller crystals or at low initial dislocation densities, the simulation data mostly oscillates around a constant value since no dislocation multiplication or cyclic hardening is observed. These results show the correlation between the flow strength, cyclic hardening, and the dislocation density evolution.

\subsection{Microstructure Evolution}

As discussed earlier, an interesting observation from the current simulations is the formation of dislocation cell structures in larger crystals. These dislocation cell structures have been previously observed experimentally in larger crystals [51] and at low strains $[44,45,46]$. Furthermore, recent continuum dislocation dynamics studies have also shown the emergence of self organized cell like dislocation structures [52]. The current simulations show that the dislocation density buildup is irreversible. This is also clear from the relative entropy plots shown in Figure 7, where the relative entropy is monotonically increasing. One measure for the strength of a dislocation network is the number of pinning points present in the system at any instance of time. Here, a pinning point is a dislocation node that is connected to at least three dislocation segments that lie on three different slip planes. This point is effectively incapable of moving due to the glide constraints associated with its dislocation arms. Figure 8(d) shows the number of pinning points versus the number of cycles for the first few cycles of different crystal sizes and initial dislocation densities. It can be observed that larger crystals develop a significant number of pinning points even after a few cycles, while smaller crystals, especially at lower densities, do not, even after 80 cycles.

The mechanisms discussed in Section 4.1, by which the dislocation density increases in larger crystals, partially explain the significant increase in the number of pinning points in larger crystals. As dislocations collide, they form pinning points, become less mobile and act as barriers for subsequent dislocation activity. In addition, intersection cross-slip, which takes place when a screw dislocation collides with a forest dislocation, becomes more frequent $[32,33,23]$. Intersection cross-slip requires less activation energy and it is more likely to take place than bulk cross-slip, which requires a high activation energy [23]. Cross-slip changes the glide plane of dislocations making them subject to further collisions with less mobile 
dislocations threading different glide planes, which subsequently results in further cross-slip and pinning point creation. Cross-slip, which is a thermally activated and a stress assisted process, is further promoted by the high local stresses in high dislocation density regions. By comparing Figures $8(\mathrm{~b})$ and $8(\mathrm{~d})$, there is an obvious one-to-one correlation between the number of pinning points and the number of cross-slip events.

The cell size quantification method discussed in Section 2.2 is utilized to analyze the dislocation cell structures emerging in the current DDD simulations. Figure 11 shows the box-count plots for crystals of different sizes, initial dislocation densities and number of cycles from a representative number of simulations that showed dislocation cell structure formation. The dislocation cell wall thicknesses are $0.03 \mu \mathrm{m}, 0.1 \mu \mathrm{m}$, and $0.15 \mu \mathrm{m}$, while the dislocation cell sizes are $0.6 \mu \mathrm{m}, 1.6 \mu \mathrm{m}$, and $2.5 \mu \mathrm{m}$, for the $2.0 \mu \mathrm{m}, 5.0 \mu \mathrm{m}$, and 7.5 $\mu \mathrm{m}$ crystals, respectively. Both the dislocation wall thickness and the dislocation cell size do not change with increasing number of cycles for the number of cycles simulated.

The box-count of small and medium sized boxes increases with increasing the number of cycles while that of bigger boxes remains the same. This indicates that the number of dislocation walls increases, but their thickness and the dislocation cell size remain constant. For this to happen, the previously formed dislocation cells are not refined by developing new dislocation walls that run across them. Alternatively, new dislocation walls develop external to the fully developed cells. Indeed, an inspection of the dislocation microstructure reveals that the complexity of the dislocation structure grows over time as can be seen in the supplementary movie II. Figure 12 shows two TEM-like slices having thickness of $0.25 \mu \mathrm{m}$ and normals (111) and (11) that were extracted from the center of the simulations shown in movie II at the end of the simulation. The dislocation free regions, which constitute the interior of the dislocation cells, are marked by the pink circles (roughly spherical in shape in $3 \mathrm{D}$ with a mean cell radius of $1.6 \mu \mathrm{m})$.

It should be noted that TEM images of the dislocation microstructure extracted from a cyclically loaded prestrained (to 0.4 strain) bulk copper single crystals oriented for multislip after 50 cycles show the formation of dislocation cell structures with dislocation cell sizes in the range of $0.7 \mu \mathrm{m}$ [53] and the mean dislocation wall thickness is $0.1 \mu \mathrm{m}$. It was 
also shown that the dislocation cell size and wall thickness both decrease with increasing prestraining level. These experimentally observed dislocation cell sizes are smaller that those predicted from those predicted from the current simulations for the $D=7.5 \mu \mathrm{m}$, which can be attributed to the much lower initial dislocation density than those in those experiments. Nevertheless, further simulations with higher initial dislocation densities are needed to investigate the effect of prestraining on the dislocation microstructure formation.

In addition, a different approach by which a multitude of information regarding the spatial distribution of dislocation structures can be obtained is by using n-point correlation functions [54]. In this approach, the simulation volume is divided into sub-volumes and the correlations between the dislocation distribution inside each sub-volume are calculated. A one- and two-point correlation functions $\alpha_{i j}(r)$ and $\beta_{i j k l}(r)$ are defined by

$$
\begin{gathered}
\alpha_{i j}(r)=\frac{1}{\Delta V} \Sigma_{p=1}^{N_{c}} \delta\left(\left\|r_{p}\right\|-r\right) \Sigma_{u=1}^{N_{p}} t_{i}^{u} b_{j}^{u} \\
\beta_{i j k l}(r)=\frac{1}{\Delta V} \Sigma_{p=1}^{N_{c}} \Sigma_{q=1}^{N_{c}} \delta\left(\left\|r_{p}-r_{q}\right\|-r\right) \Sigma_{u=1}^{N_{p}} \Sigma_{v=1}^{N_{q}} t_{i}^{u} b_{j}^{u} t_{k}^{v} b_{l}^{v}
\end{gathered}
$$

where $\Delta V$ is the computation cell volume, $N_{c}$ is the number of sub-volumes in the simulation cell, $r_{p}$ and $r_{q}$ are the position vectors of the centers of the sub-volumes $p$ and $q$ respectively, $N_{p}$ and $N_{q}$ are the numbers of dislocation segments in the sub-volumes $p$ and $q$, respectively, $\delta$ is the Dirac delta function, $t_{i}^{u}$ is the line vector of the $u^{t h}$ dislocation segment, and $b_{j}^{u}$ is its Burgers vector. Higher order n-point correlation functions can also be constructed in the same manner by summing over every combination of $n$ sub-volumes. The two-point correlation function is of interest here since the locations of the peaks of this function give the separation distance of regions of high dislocation density, which in the current simulations are the dislocation walls. The two-point correlation, defined by Equation (4), is a set of 81 distributions that fully describe the spatial distribution of the dislocation structure along with the Burgers vector distribution. For the purposes of this analysis, a reduced data set is used where the dislocations of different Burgers vectors are lumped together. This gives a two-point dislocation density vector defined by 


$$
\rho_{i j}(r)=\beta_{i p j q}(r) O_{p q}
$$

where $O_{p q}=1$ for all $p$ and $q$. This is a set of 9 distributions only. The components of the two-point density vector for the final dislocation microstructure of three crystals of size $D=5.0 \mu \mathrm{m}$ and $\rho_{o}=10^{12} \mathrm{~m}^{-2}$, are showin in Figure 13. The spacing between the peaks in the two-point correlations, which corresponds to the average dislocation cell size, ranges between $1.4 \mu \mathrm{m}$ and $1.7 \mu \mathrm{m}$. This agrees with the box-counting analysis discussed earlier in the dislocation cell size estimate of $1.6 \mu \mathrm{m}$. It should be noted however that the twopoint correlation is more sensitive to other dislocation distribution features, for example, the spacing distance between two adjacent dislocation cell walls corresponds to a smaller peak because the dislocation walls do not consist of perfectly parallel dislocation segments. This explains the wider peaks in the two-point correlations. 


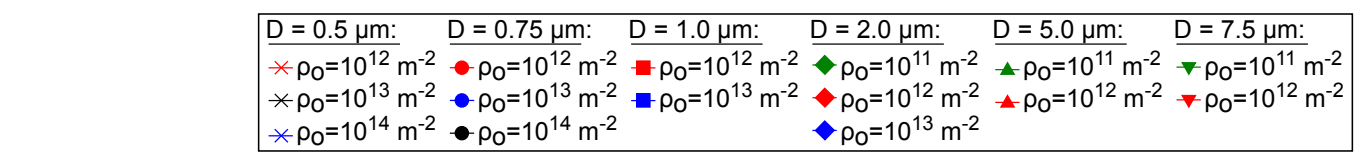

(a)

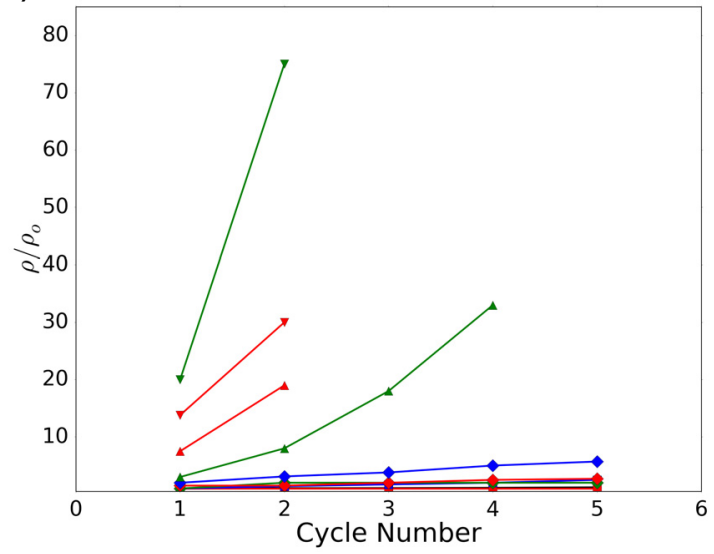

(c)

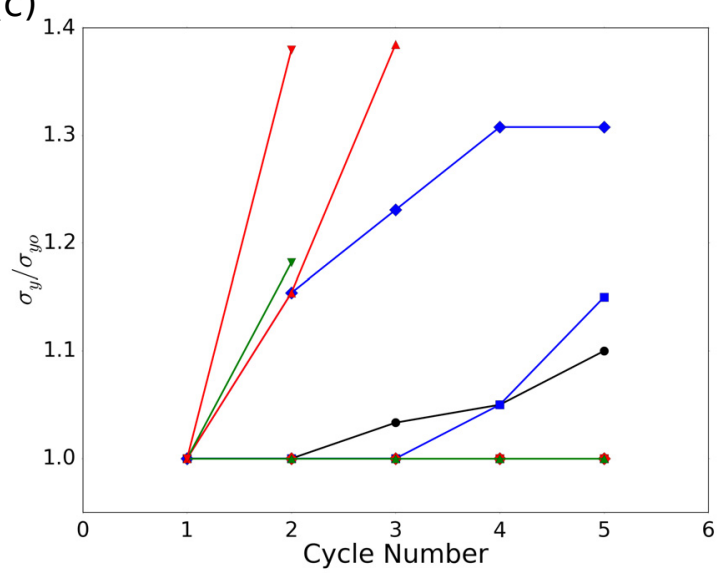

(b)

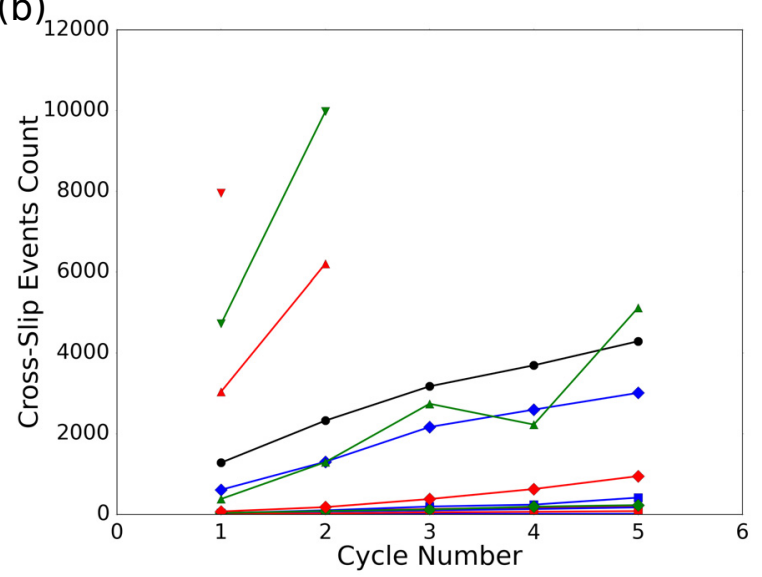

(d)

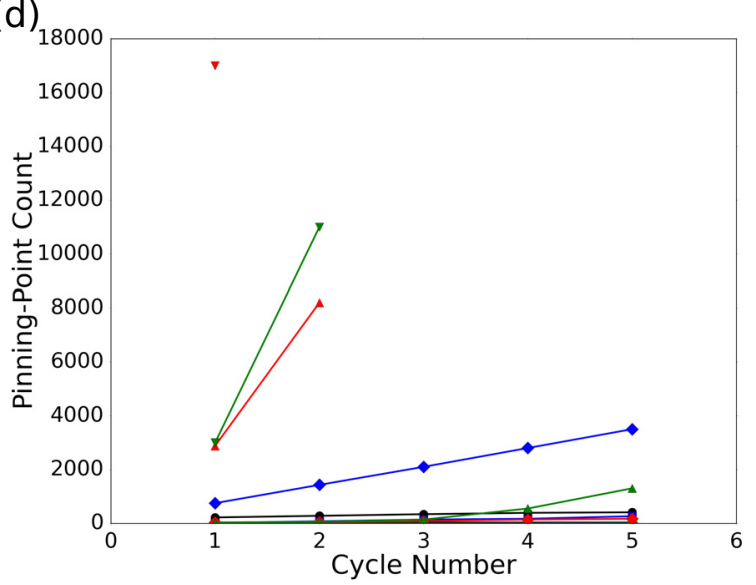

Figure 8: (a) Dislocation density multiplication factor; (b) number of cross-slip events; (c) relative flow strength; and (d) number of dislocation pinning points, as a function of the number of loading cycle for different simulation conditions. Each data point is an average of three different simulations with varying initial random dislocation distributions. 


\begin{tabular}{|c|c|c|}
\hline $\begin{array}{l}n=[111] B=1 / 2[1 \overline{1} 0] \\
n=[111] B=1 / 2[10 \overline{1}] \\
n=[111] B=1 / 2[01 \overline{1}]\end{array}$ & 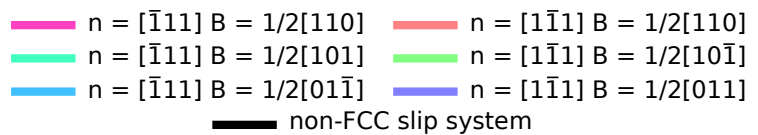 & $\begin{array}{l}n=[11 \overline{1}] B=1 / 2[1 \overline{1} 0] \\
n=[11 \overline{1}] B=1 / 2[101] \\
n=[11 \overline{1}] B=1 / 2[011]\end{array}$ \\
\hline
\end{tabular}
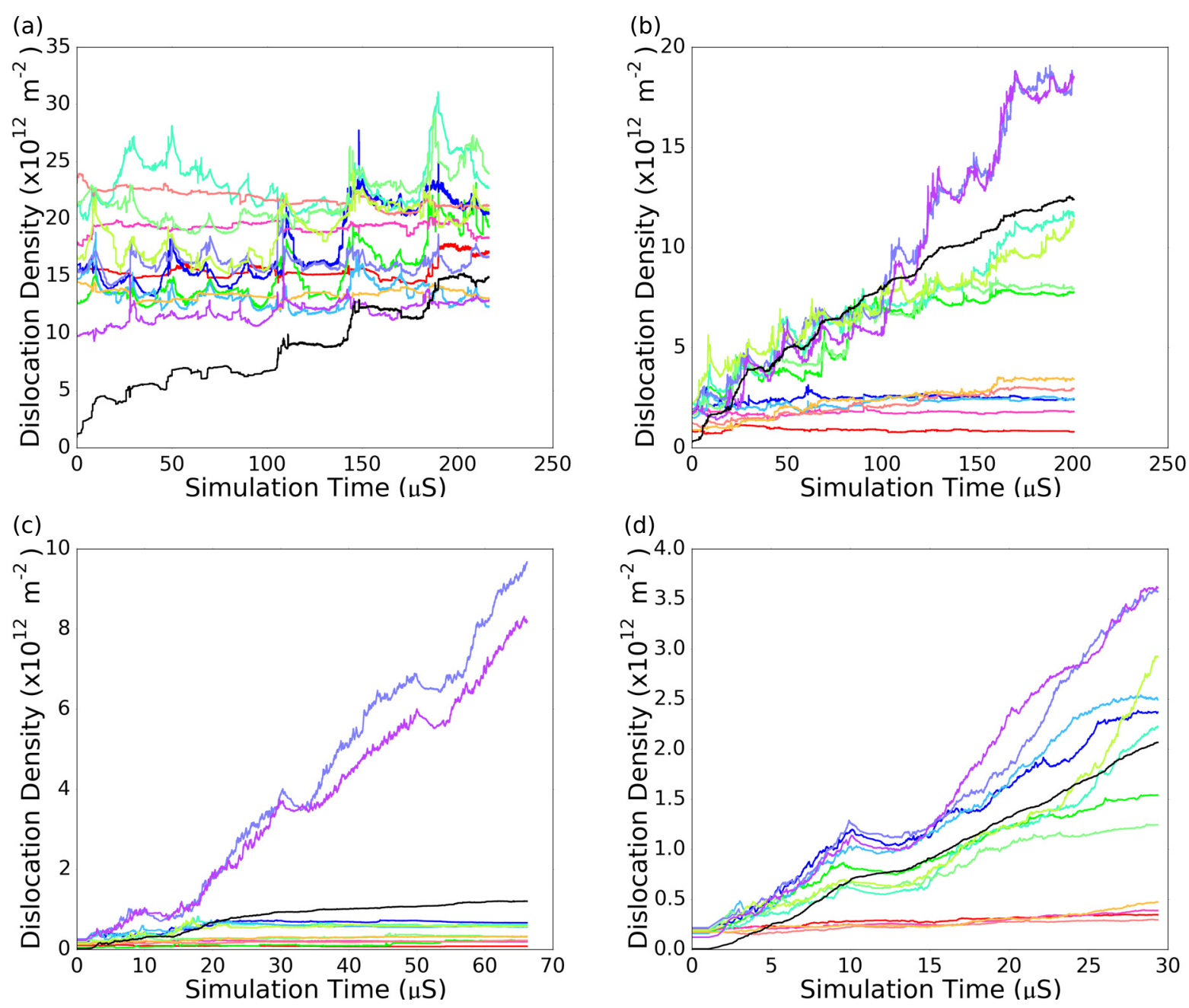

Figure 9: The evolution of dislocation density per slip system as a function of simulation time for different crystal sizes and initial dislocation densities: (a) $D=0.75 \mu \mathrm{m}$ and $\rho_{o}=10^{14} \mathrm{~m}^{-2}$; (b) $D=2.0 \mu \mathrm{m}$ and $\rho_{o}=10^{13} \mathrm{~m}^{-2}$; (c) $D=5.0 \mu \mathrm{m}$ and $\rho_{o}=10^{12} \mathrm{~m}^{-2}$; and (d) $D=7.5 \mu \mathrm{m}$ and $\rho_{o}=10^{12} \mathrm{~m}^{-2}$. 


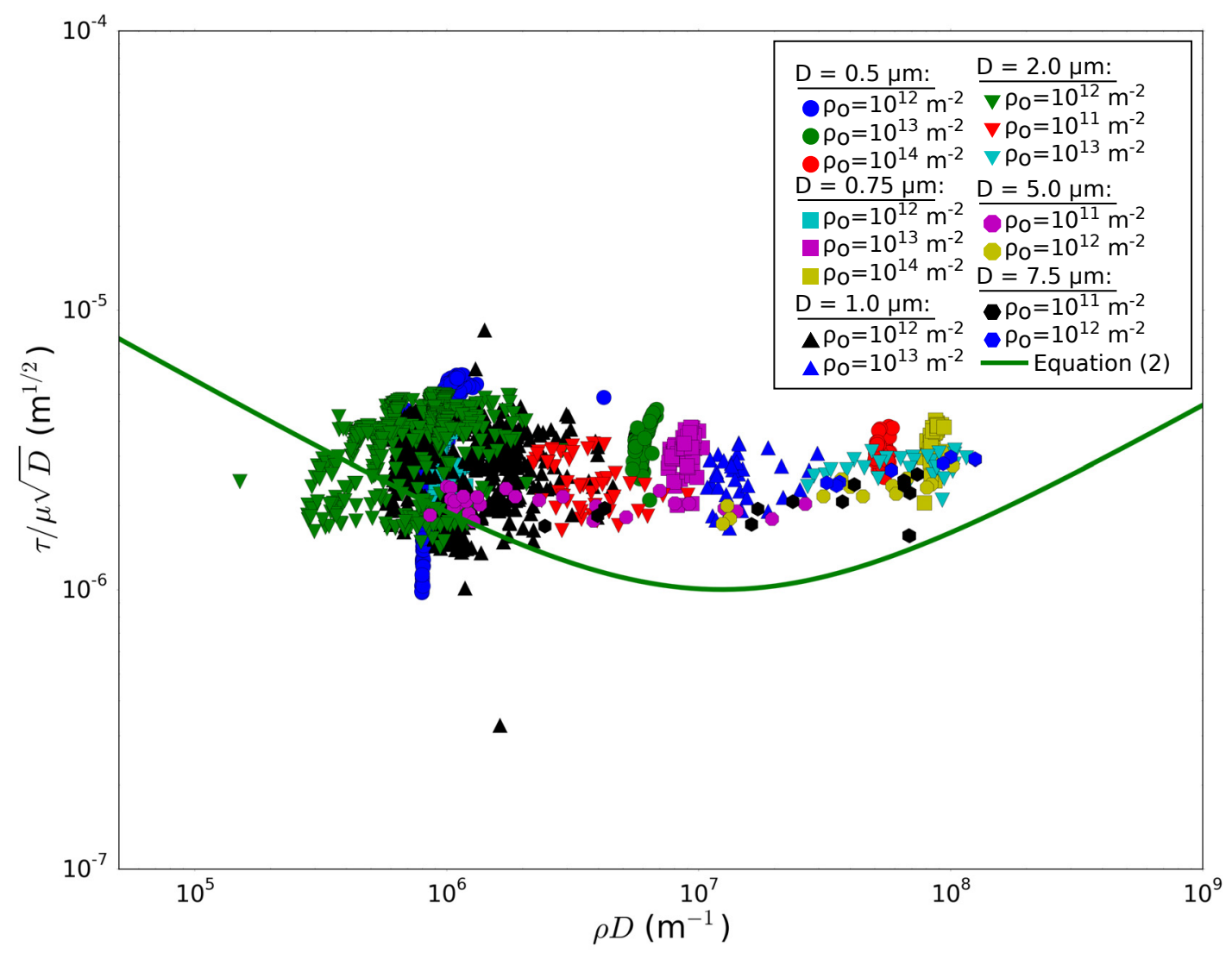

Figure 10: The flow strength normalized by the shear modulus and multiplied by the square root of the crystal size versus the dislocation density multiplied by the crystal size at the end of each quarter loading cycle from all the simulations. 

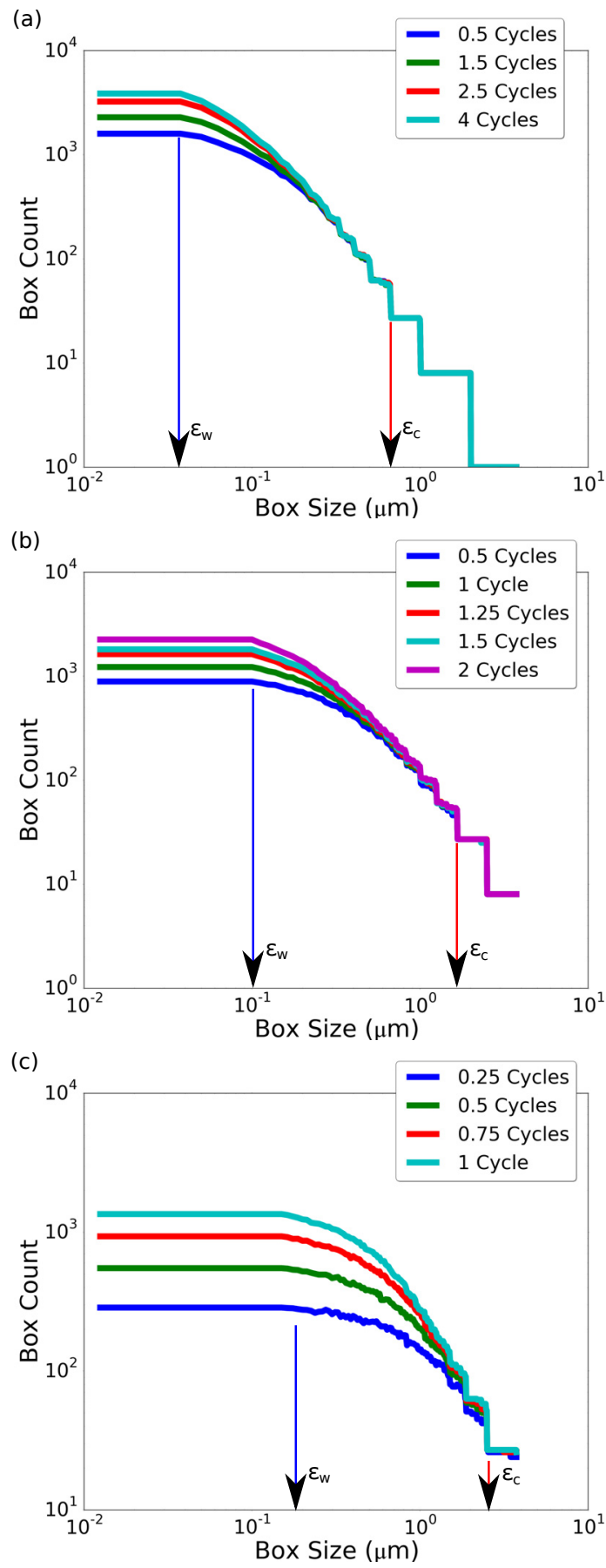

Figure 11: The box-counting method applied to representative dislocation microstructures from the current DDD simulations: (a) $D=2.0 \mu \mathrm{m}$ and $\rho_{o}=10^{13} \mathrm{~m}^{-2}$; (b) $D=5.0 \mu \mathrm{m}$ and $\rho_{o}=10^{12} \mathrm{~m}^{-2}$; and (a) $D=7.5$ $\mu \mathrm{m}$ and $\rho_{o}=10^{12} \mathrm{~m}^{-2}$. The blue arrows indicate the wall thickness and the red ones indicate the cell size. 
(a)

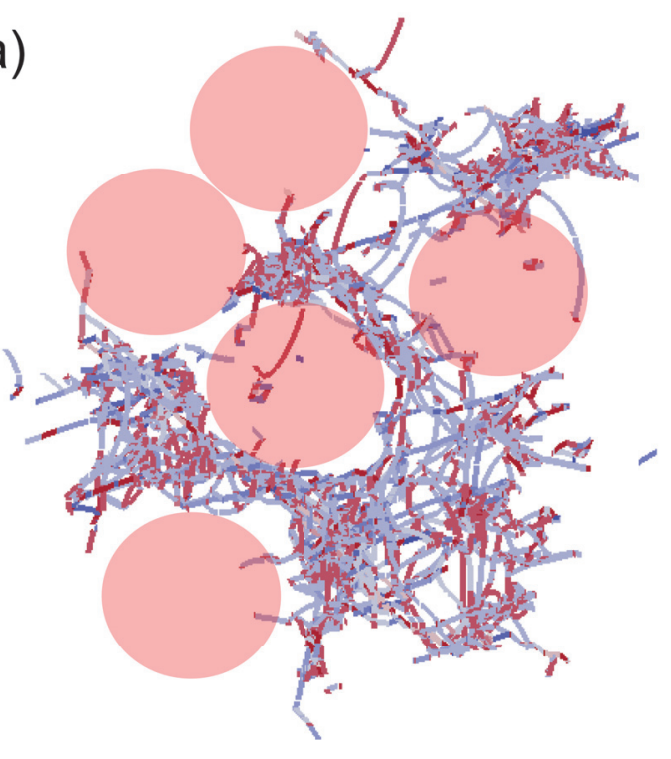

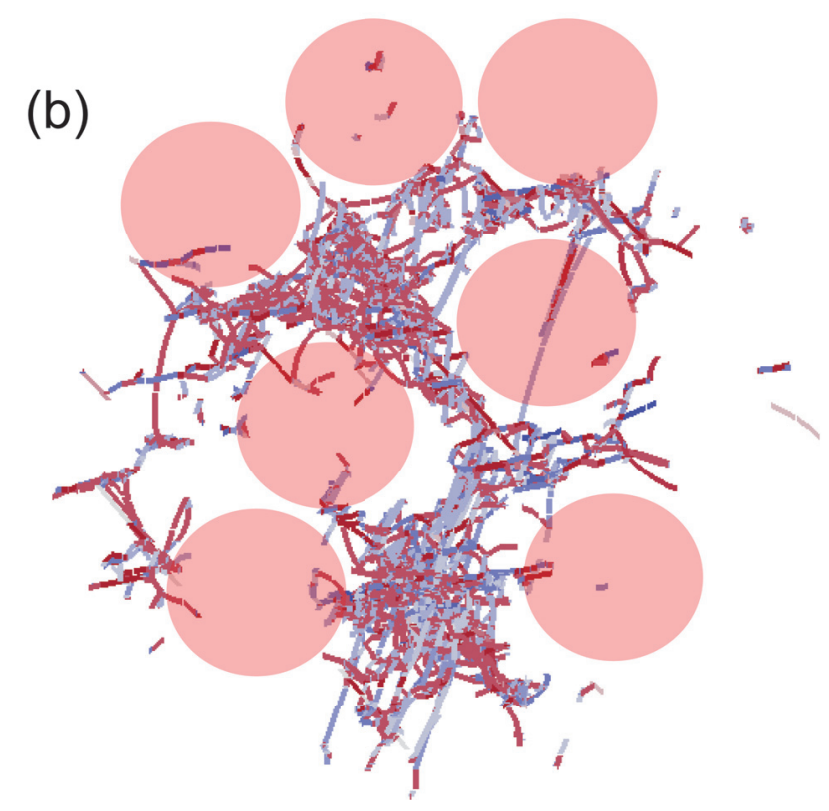

Figure 12: Two TEM-like slices having thickness of $0.25 \mu \mathrm{m}$ extracted at the end of the simulation from a $D=5.0 \mu \mathrm{m}$ simulation cell with an initial dislocation density of $\rho_{o}=10^{12} \mathrm{~m}^{-2}$. The dislocation cells and dislocation cell walls can be clearly observed and the low dislocation density cell interiors are marked by pink circles: (a) a slice along the (111) plane; and (b) a slice along the (111) plane. The average cell size is about $1.6 \mu \mathrm{m}$. 

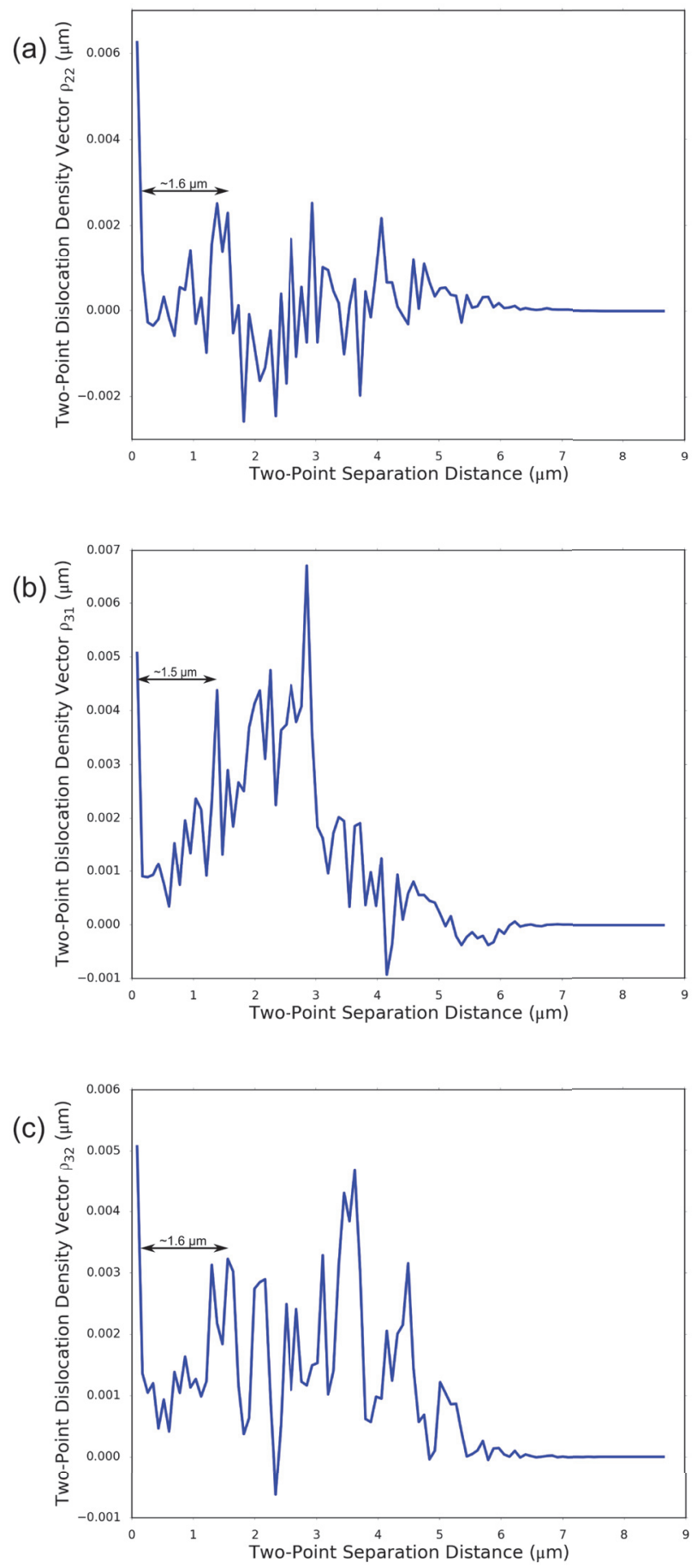

Figure 13: Selected two-point density vector plots for the final dislocation microstructure of three crystals having size $D=5.0 \mu \mathrm{m}$ and an initial dislocation density of $\rho_{o}=10^{12} \mathrm{~m}^{-2}$ : (a) $\rho_{11}$ for the first crystal; (b) $\rho_{31}$ for the second crystal; and (c) $\rho_{32}$ for the third crystal. The distances between the first two peaks are shown. 


\section{Summary and Conclusions}

The mechanical response and dislocation microstructure evolution of single crystal FCC Nickel during mechanical cyclic loading have been studied using three-dimensional discrete dislocation dynamics simulations. The dependence of the cyclic strength on crystal size and initial dislocation density has been analyzed. It was found that larger crystals show cyclic hardening, significant dislocation density multiplication and dislocation structure evolution. The evolution of the dislocation microstructures was attributed to the buildup of pinning points in the dislocation network, a behavior which correlates one-to-one with the frequency of cross-slip events. A metric was suggested and utilized to study the extent to which a dislocation microstructure would evolve into a dislocation cell structure over time. It was found that larger crystals develop early fatigue dislocation cell structures that do not break down with load reversal. Finally, the box-counting method was used to estimate the thickness of the dislocation walls and the size of the dislocation cells and it was found that they depend on the crystal size but not on the number of loading cycles.

\section{Acknowledgements}

This research was sponsored by DARPA contract number N6600112-14229 and in part by the Army Research Laboratory through contract number W911NF-12-2-0022. Part of the calculations were performed on resources at the DoD HPCMP Open Research Systems.

\section{About the Authors}

\section{Ahmed M. Hussein}

Ahmed Hussein is a $\mathrm{PhD}$ candidate in the department of Mechanical Engineering at Johns Hopkins University studying the plasticity and crack initiation in metallic materials through large scale simulations of their dislocation microstructures. He received his B.Sc and M.Sc from the Aerospace Engineering Department in Cairo University, Egypt, in 2008 and 2010 respectively. Before starting his graduate studies, he worked as a software developer for Applied Science Intl. developing geometry processing and material models libraries for 


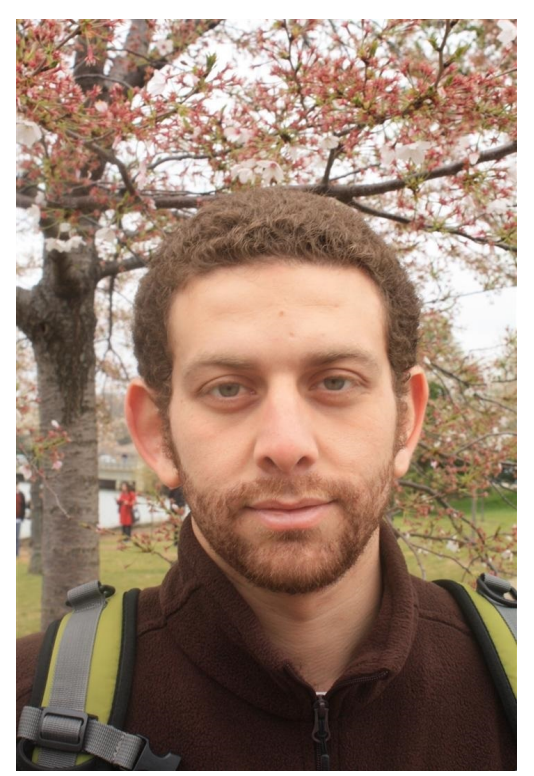

engineering analysis software used in simulating the behavior of structures under extreme loading conditions.

Dr. Jaafar A. El-Awady

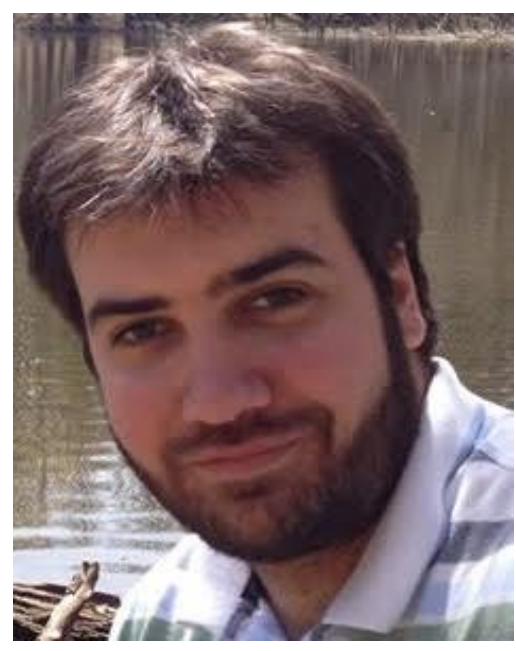

Dr. El Awady is an Assistant Professor of Mechanical Engineering at Johns Hopkins University since 2010. His research focuses on developing multiscale simulations and microscale experiments to predict the underlying deformation, damage, and failure mechanisms in materials. He received his B.S. and M.S. from Cairo University, Egypt, and his Ph.D. from 
the University of California-Los Angles, all in Aerospace Engineering. Prior to joining JHU, he was a visiting scientist at the Wright Patterson Air Force Research Laboratory. He is the recipient of multiple awards including: DARPA Young Investigator (2012), ASME Orr Early Career (2014), and National Science Foundation CAREER (2015).

\section{References}

[1] R.I. Stephens, A. Fatemi, R.R. Stephens, and H.O. Fuchs. Metal Fatigue in Engineering, 2nd Ed. Wiley, 2000.

[2] J. Schijve. Fatigue damage in aircraft structures not wanted but tolerated. Int. J. Fatigue, 31:998-1011, 2009.

[3] H. Mughrabi. Fatigue, an everlasting materials problem - still en vogue. Proc. Engineering, 2:3-26, 2010.

[4] S. Suresh. Fatigue of Materials, 2nd Ed. Cambridge University Press, 1998.

[5] H. Mughrabi and H.W. Hoppel. Cyclic deformation and fatigue properties of very fine-grained metals and alloys. Int. J. Fatigue, 32:1413-1427, 2010.

[6] B. Bako and I. Groma. Stochastic approach for modeling dislocation patterning. Phys. Rev. B, 60(1):122-127, 1999.

[7] I. Groma and B. Bako. Dislocation patterning: From micro- to mesoscale description. Phys. Rev. Lett., 84(7):1487 - 1490, 2000.

[8] M. Koslowski R. Thomson and R. LeSar. Modeling energetics and noise in dislocation patterning. Phys. Rev. B, 73, 2006.

[9] L.P. Kubin, G. Canova, M. Condat, B. Devincre, V. Pontikis, and Y. Bréechet. Dislocation microstructures and plastic flow: a 3-D simulation. Solid State Phenom., 23-24:455-472, 1992.

[10] N.M. Ghoniem, S.-H. Tong, and L.Z. Sun. Parametric dislocation dynamics: A thermodynamics-based approach to investigations of mesoscopic plastic deformation. Phys. Rev. B, 61(2):913-927, 2000.

[11] H.M. Zbib, M. Rhee, and J.P. Hirth. A multiscale model of plasticity. Int. J. Plasticity, 18:11331163, 2002.

[12] D. Weygand, L.H. Friedman, E. Van der Giessen, and A. Needleman. Aspects of boundary-value problem solutions with three-dimensional dislocation dynamics. Mod. Sim. Mater. Sci. Eng., 10(4):437$468,2002$.

[13] C. Motz, D. Weygand, J. Senger, and P. Gumbsch. Initial dislocation structures in 3-D discrete dislocation dynamics and their influence on microscale plasticity. Acta Mater., 57(6):1744-1754, 2009. 
[14] J.A El-Awady. Unravelling the physics of size-dependent dislocation-mediated plasticity. Nature Comm., 6, 2015.

[15] P. Pant, K.W. Schwarz, and S.P. Baker. Dislocation interactions in thin FCC metal films. Acta Mater., 51:3243-3258, 2003

[16] M.C. Fivel, C.F. Robertson, G.R. Canova, and L. Boulanger. Three-dimensional modeling of indentinduced plastic zone at a mesoscale. Acta Mater, 46:6183-6194, 1998.

[17] T.A. Khraishi, H.M. Zbib, T.D. de la Rubia, and M. Victoria. Modelling of irradiation-induced hardening in metals using dislocation dynamics. Phil. Mag. Lett., 81:583-593, 2001.

[18] V.S. Deshpande, A. Needleman, and E. Van der Giessen. Discrete dislocation plasticity modeling of short cracks in single crystals. Acta Mater., 51:1-15, 2003.

[19] S. Brinckmann and Van der Giessen E. A discrete dislocation dynamics study aiming at understanding fatigue crack initiation. Mat. Sci. Eng. A, 387-389:461-464, 2004.

[20] C. Déprés, C.F. Robertson, and M.C. Fivel. Low strain fatigue in 316L steel surface grains: a threedimensioin discrete dislocation dynamics modeling of the early cycles. Part 1: Dislocation microstructures and mechanical behavior. Phil. Mag., 84(22):2257-2275, 2004.

[21] C. Déprés, C.F. Robertson, and M.C. Fivel. Low strain fatigue in 316L steel surface grains: a threedimensioin discrete dislocation dynamics modeling of the early cycles. Part 2: Persistent slip markings and micro-crack nucleation. Phil. Mag., 86(1):79-97, 2006.

[22] J.A. El-Awady, N.M. Ghoniem, and H. Mughrabi. Dislocation modelling of localized plasticity in persistant slip bands. In B.L. Adams and A. Garmestani, editors, Proceedings of the 136th TMS Annual Meeting and Exhibition, pages 23-35, 2007.

[23] A.M. Hussein, S.I. Rao, M.D. Uchic, D.M. Dimiduk, and J.A. El-Awady. Microstructurally based cross-slip mechanisms and their effects on dislocation microstructure evolution in fcc crystals. Acta Mater., 85:180-190, 2015.

[24] A. Arsenlis, W. Cai, M. Tang, M. Rhee, T. Oppelstrup, G. Hommes, T.G. Pierce, and V.V. Bulatov. Enabling strain hardening simulations with dislocation dynamics. Mod. Sim. Mater. Sci. Eng., 15(6):553-595, 2007.

[25] E.Y. Gutmanas and Nadgornyi E.M. Dislocation multiplication by multiple cross slip. Sov. Phys. Solid State, 12:733-734, 1970.

[26] P.J. Jackson. The role of cross-slip in the plastic deformation of crystals. Mater. Sci. Eng., 57:39-47, 1983.

[27] P.J. Jackson. Dislocation modelling of shear in FCC crystals. Prog. Mater. Sci., 29(1-2):139-175, 1985.

[28] G. Saada. Cross-slip and work hardening of fcc crystals. Mater. Sci. Eng. A., 137:177-183, 1991.

[29] K. Jumonji, S. Ueta, A. Miyahara, M. Kato, and A. Sato. Rapid work hardening caused by cube cross 
slip in $\mathrm{Ni}_{3} \mathrm{Al}$ single crystals. Phil. Mag. A, 73:345-364, 1996.

[30] R. Madec, B. Devincre, and L.P. Kubin. Simulation of dislocation patterns in multislip. Scripta Mater., 47(10):689-695, 2002.

[31] L.P. Kubin, B. Devincre, and T. Hoc. Inhibited dynamic recovery and screw dislocation annihilation in multiple slip of fcc single crystals. Phil. Mag., 86:4023-4036, 2006.

[32] S.I. Rao, D.M. Dimiduk, J.A. El-Awady, T.A. Parthasarathy, M.D. Uchic, and C. Woodward. Atomistic simulations of athermal cross-slip nucleation at screw dislocation intersections in face-centered cubic nickel. Phil. Mag., 89(34-36):3351-3369, 2009.

[33] S.I. Rao, D.M. Dimiduk, J.A. El-Awady, T.A. Parthasarathy, M.D. Uchic, and C. Woodward. Activated states for cross-slip at screw dislocation intersections in face-centered cubic nickel and copper via atomistic simulation. Acta Mater., 58:5547-5557, 2010.

[34] S.I. Rao, D.M. Dimiduk, T.A. Parthasarathy, J. El-Awady, Woodward C., and M.D. Uchic. Calculations of intersection cross-slip activation energies in fcc metals using nudged elastic band method. Acta Mater., 59(19):7135 - 7144, 2011.

[35] S.I. Rao, D.M. Dimiduk, J.A. El-Awady, T.A. Parthasarathy, M.D. Uchic, and C. Woodward. Spontaneous athermal cross-slip nucleation at screw dislocation intersections in fcc metals and L12 intermetallics investigated via atomistic simulations. Phil. Mag., 93(22):3012-3028, 2013.

[36] S.I. Rao, D.M. Dimiduk, T.A. Parthasarathy, M.D. Uchic, and C. Woodward. Atomistic simulations of surface cross-slip nucleation in face-centered cubic nickel and copper. Acta Mater., 61(7):2500-2508, 2013.

[37] C. Zhou and R. Lesar. Dislocation dynamics simulations of plasticity in polycrystalline thin films. Int. J. Plasticity, 30-31:185-201, 2012.

[38] H. Fan, S. Aubry, A. Arsenlis, and J. El-Awady. The role of twinning deformation on the hardening response of polycrystalline magnesium from discrete dislocation dynamics simulations. Acta Mat., 92:126-139, 2015.

[39] C.E. Shannon. A mathematical theory of communication. Bell syst. tech. J., 27(3):379-423, 1948.

[40] M. Zaiser, K. Bay, and P. Hähner. Fractal analysis of deformation-induced dislocation patterns. Acta Mater., 47(8):2463 - 2476, 1999.

[41] A.M. Hussein and J.A. El-Awady. Surface roughness evolution during early stages of mechanical cyclic loading. Int. J. Fatigue, 87:339-350, 2016.

[42] H. Mughrabi, T. Ungar, W. Kienle, and M. Wilkens. Long-range internal stresses and asymmetric x-ray line-broadening in tensile-deformed [001]-orientated copper single crystals. Phil. Mag., 53(6):793-813, 1986.

[43] J. Cuddy and N. Bassim. Study of dislocation cell structures from uniaxial deformation of aisi 4340 
steel. Mat. Sci. Eng. A, 113:421-429, 1989.

[44] B. Jakobsen, U. Lienert, J. Almer, W. Pantleon, and H.F Poulsen. Properties and dynamics of bulk subgrains probed in-situ using a novel x-ray diffraction method. Mat. Sci. Forum, 550:613-618, 2007.

[45] D.M. Norfleet, D.M. Dimiduk, S.J. Polasik, M.D. Uchic, and M.J. Mills. Dislocation structures and their relationship to strength in deformed nickel microcrystals. Acta Mater., 56(13):2008, 2008.

[46] P. Landau, R.Z. Shneck, G. Makov, and A. Venkert. Evolution of dislocation patterns in fcc metals. IOP Conf. Ser.: Mater. Sci. Eng, 3, 2009.

[47] H. Mughrabi. The cyclic hardening and saturation behaviour of copper single crystals. Mater. Sci. Eng., 33:207-223, 1978.

[48] C.D. Liu, D.X. You, and M.N. Bassim. Cyclic strain hardening in polycrystalline copper. Acta Metall. Mater., 42(5):1631-1638, 1993.

[49] D.J. Morrison and Chopra V. Cyclic stress-strain response of polycrystalline nickel. Mater. Sci. Eng. A, 177:29-42, 1994.

[50] N.Y Jin and A.T. Winter. Role of cross-slip of screw dislocations in fatigue behavior of copper single crystals. in Basic Questions in Fatigue, Vol I. pages 17-25, 1988.

[51] Q. Yu, R.K. Mishra, J.W. Morris, and A.M. Minor. The effect of size on dislocation cell formation and strain hardening in aluminum. Phil. Mag., 94:2062-2071, 2014.

[52] Y.S. Chen, W. Choi, S. Papanikolaou, M. Bierbaum, and Sethna J.P. Scaling theory of continuum dislocation dynamics in three dimensions: Self-organized fractal pattern formation. Int. J. Plasticity, 46:94-129, 2013.

[53] J. Schrank, W. Prantl, and Stuwe H.P. The influence of crystal orientation on the fatigue softening behaviour of severely predeformed copper. Mat. Sci. Eng. A, 110:61-68, 1989.

[54] E. Kroener. Initial studies of a plasticity theory based upon statistical mechanics. in Inelastic Behaviour of Solids. pages 137-147, 1969. 